\title{
Comparative Analysis of Glycogene Expression in Different Mouse Tissues Using RNA-Seq Data
}

\author{
Ahmad Firoz, ${ }^{1,2}$ Adeel Malik, ${ }^{3}$ Sanjay Kumar Singh, ${ }^{2,4}$ Vivekanand Jha,, ${ }^{2,5}$ and Amjad Ali ${ }^{1}$ \\ ${ }^{1}$ School of Chemistry and Biochemistry, Thapar University, Patiala, Punjab 147004, India \\ ${ }^{2}$ Biomedical Informatics Center of ICMR, Post Graduate Institute of Medical Education and Research (PGIMER), \\ Chandigarh 160012, India \\ ${ }^{3}$ School of Biotechnology, Yeungnam University, 280 Daehak-ro, Gyeongsan, Gyeongbuk 712-749, Republic of Korea \\ ${ }^{4}$ Department of Biotechnology, Thapar University, Patiala, Punjab 147004, India \\ ${ }^{5}$ Department of Nephrology, Post Graduate Institute of Medical Education and Research (PGIMER), Chandigarh 160012, India
}

Correspondence should be addressed to Ahmad Firoz; ahmadfirozbin@gmail.com and Adeel Malik; adeel@procarb.org

Received 13 May 2014; Accepted 10 June 2014; Published 9 July 2014

Academic Editor: Giulia Piaggio

Copyright ( 2014 Ahmad Firoz et al. This is an open access article distributed under the Creative Commons Attribution License, which permits unrestricted use, distribution, and reproduction in any medium, provided the original work is properly cited.

\begin{abstract}
Glycogenes regulate a wide array of biological processes in the development of organisms as well as different diseases such as cancer, primary open-angle glaucoma, and renal dysfunction. The objective of this study was to explore the role of differentially expressed glycogenes (DEGGs) in three major tissues such as brain, muscle, and liver using mouse RNA-seq data, and we identified 579, 501, and 442 DEGGs for brain versus liver (BvL579), brain versus muscle (BvM501), and liver versus muscle (LvM442) groups. DAVID functional analysis suggested inflammatory response, glycosaminoglycan metabolic process, and protein maturation as the enriched biological processes in BvL579, BvM501, and LvM442, respectively. These DEGGs were then used to construct three interaction networks by using GeneMANIA, from which we detected potential hub genes such as PEMT and HPXN (BvL579), IGF2 and NID2 (BvM501), and STAT6 and FLT1 (LvM442), having the highest degree. Additionally, our community analysis results suggest that the significance of immune system related processes in liver, glycosphingolipid metabolic processes in the development of brain, and the processes such as cell proliferation, adhesion, and growth are important for muscle development. Further studies are required to confirm the role of predicted hub genes as well as the significance of biological processes.
\end{abstract}

\section{Introduction}

Cell function within an organism has huge variation due to gene expression pattern although all cells in an individual mammal have almost identical DNA [1]. It is important to know how cells and tissues differ in gene expression which is regulated during developmental changes in different tissues, consequently affecting their biological functions. The pattern of glycogene expression is one of important factors that provide clues about many biological functions, developmental changes, and diseases in human, mouse, and tissues of other organisms [2-4]. Glycogene is a gene that is responsible for the glycosylation of proteins, lipids, and proteoglycans and includes genes associated with the synthesis of glycans such as glycosyltransferase, sugar-nucleotide transporters, and sulfotransferases $[5,6]$. Similarly, the term glycogenome involves all genes that play a role in glycosylation and represents more than 600 genes in the mouse genome [7]. Glycosylation is one of the most common posttranslational modification reactions [8]. The glycogene expression is relatively weak as compared to other molecules; however, different glycogenes are upregulated by specific tissues depending on the local conditions [5].

In order to understand the pathology of various diseases, recently, many studies have targeted and explored the role of glycogenes expression in various diseases [9-12]. Alteration in the glycogene expression is also associated with drug resistance of various types of cancers including pancreatic 
cancer epithelial-mesenchymal transition [13], breast cancer [14], and hepatocarcinoma [15]. The change in the glycogene expression is also associated with conjunctival epithelium [2] and primary open-angle glaucoma (POAG) [16]. Moreover, the defects in the normal expression of glycogenes in tonsillar B lymphocytes are also correlated with the proteinuria and renal dysfunction in IgA nephropathy $[17,18]$.

Several high-throughput microarray studies have been applied to investigate the functional roles of various glycogenes $[11,19,20]$. However, to the best of our knowledge, no study till date has utilized RNA-Seq data in the existing databases to specifically explore the role of glycogenes. Previously, we identified the role of glycogenes in skeletal muscle development in MYOG $_{\mathrm{kd}}$ cells by using RNA-Seq data. Therefore, in this work, we intend to investigate the role of glycogenes in various biological processes that are involved in the development of brain, muscle, and liver tissues using the precomputed expression values from mouse RNAseq data. Functional and pathway analysis of differentially expressed glycogenes (DEGGs) between BvL579, BvM501, and LvM442 were enriched with gene ontology (GO) terms and Kyoto Encyclopedia of Genes and Genomes (KEGG) pathways, respectively. Additionally, the interaction networks of DEGGs were constructed to identify the hub genes as well as to detect the functional modules in these networks by using community analysis. We expect that the findings of our study may shed new lights on the roles of glycogenes in the development of these tissues and their pathogenesis.

\section{Materials and Methods}

2.1. Datasets. The precomputed expression values with no spike for the brain, liver, and muscles C57BL6 mouse tissues were downloaded from WoldLab (http://woldlab.caltech .edu/ alim/RNAseq/) for our computational analysis. These precomputed expression values represent the RNA-seq analysis initially performed by Mortazavi et al., using Illumina Genome Analyzer and expression values computed by using ERANGE package [21]. To remove low expressed genes the dataset was filtered by removing genes with RPKM value $<1$ in all the three tissues.

2.2. Fold Change Analysis. Fold change is often used in analysis of gene expression data of RNAseq experiments, for measuring change in the expression level of a gene and comparing the expression of genes between two sets of arrays, for example, case and control sets. Fold change analysis of genes commonly expressed between two different samples was done to compare the gene expression in glycogenes [22, 23]. Fold change was calculated as the ratio of brain versus liver, brain versus muscle, and liver versus muscle groups. Additionally, to further investigate the role of glycogenes in different tissues, the differentially expressed genes were manually verified in the UniProt database [24] to check whether they represent a glycogene or not. A gene was considered as a glycogene if it was annotated as a "glycoprotein" in the "Keywords" section of the UniProt database.
2.3. Functional Analysis. DAVID (http://david.abcc.ncifcrf .gov/home.jsp) functional annotation cluster analysis was performed on the list of differentially expressed glycogenes (DEGGs) with a fold change of $\geq 2$. Only those terms that reported a $P$ value of $\leq 0.05$ and count number $\geq 5$ genes were selected for analysis. The gene ontology (GO) term biological process (BP) in DAVID was used to categorize enriched biological themes in the list of DEGGs. Pathway mapping was performed using the KEGG Automatic Annotation Server (KAAS) (http://www.genome.jp/kegg/kaas/) [25]. The amino acid sequences of these DEGGs were uploaded to the KAAS web server as an input using single-directional best hit $(\mathrm{SBH})$ method to assign orthologs. KAAS offers functional annotation of genes in a genome via a BLAST similarity searches against a manually curated set of ortholog groups in the KEGG GENES database. KAAS assigned a KEGG orthology $(\mathrm{KO})$ number to genes in the data sets, which were mapped to one of KEGG's reference pathways.

2.4. Network Analysis. The functional interactions between DEGGs were analyzed by GeneMANIA webserver [26]. The GO term biological process was used to create the interaction network between the DEGGs and 50 additional genes by using mouse as a source species. The relationship between the genes in the network includes coexpression, physical and genetic interactions, pathways, colocalization, protein domain similarity, and predicted interactions. The network was filtered by removing all the interactions where weights $<0.1$.

2.5. Identification of Hub Genes. Generally, the biological networks exhibit the scale-free property [27] where only a few nodes in the network have many connections that represent hubs in the network. Hub genes were identified by calculating the node degree distribution [28] by using the NetworkAnalyzer plugin of Cytoscape. A glycogene with the highest degree distribution was considered as a hub in the current network.

2.6. Community Analysis. The functional modules within the network were further detected by using the greedy community-structure detection algorithm, implemented in the Cytoscape plugin GLay (http://brainarray.mbni.med .umich.edu/sugang/glay/) [29]. GLay offers Cytoscape users a diverse group of community structure algorithms and graph layout functions for network clustering and structured visualization [29]. To identify the overrepresented biological functions within each cluster, the detected clusters were subjected to functional enrichment analyses by DAVID functional analysis tool. For enrichment analysis, only those communities were analyzed which have at least 10 nodes.

\section{Results}

3.1. Identification of Differentially Expressed Glycogenes (DEGGs). We downloaded the precomputed RPKM values for mouse transcriptome that was mapped and quantified previously by RNA-Seq analysis [21]. This RNA-Seq data 
TABLE 1: Gene expression summary.

(a) Total number of genes in each sample before and after filtering. Each tissue sample consists of 33598 genes that were filtered by removing all those genes that showed RPKM value $<1$

\begin{tabular}{lcc}
\hline Tissue & Data filtering & $\begin{array}{c}\text { Total number of genes } \\
\text { after filtering }\end{array}$ \\
\hline Brain & 33598 & 15,237 \\
Liver & 33598 & 11,920 \\
Muscle & 33598 & 12,202 \\
\hline
\end{tabular}

(b) Total number of differentially expressed genes between different tissues

\begin{tabular}{lccc}
\hline & Genes commonly expressed between different tissues \\
Tissue & $\begin{array}{c}\text { Number of expressed } \\
\text { genes }\end{array}$ & $\begin{array}{c}\text { Number of differentially } \\
\text { expressed genes (DEGs) } \\
(\geq 2 \text { fold change) }\end{array}$ & $\begin{array}{c}\text { Number of differentially } \\
\text { expressed glycogenes } \\
\text { (DEGGs) } \\
(\geq 2 \text { fold change) }\end{array}$ \\
\hline Brain versus liver & 10,653 & 5228 & 579 \\
Brain versus muscle & 10,820 & 5283 & 501 \\
Liver versus muscle & 9,833 & 3840 & 442 \\
\hline
\end{tabular}

consists of RNA from adult mouse brain, liver, and skeletal muscle tissues and represents 33598 genes in each tissue sample. The data was further filtered by excluding the genes with RPKM values $<1$ from the analysis. As a result, 15,237, 11,920 , and 12,202 genes were identified from brain, liver, and muscle samples, respectively (Table 1(a)). Then three groups (brain versus liver, brain versus muscle, and liver versus muscle) were created and the number of genes that were common in each group were identified and summarized in Table 1(b). From the table, it can be observed that the number of common genes between brain versus liver is 10653 , brain versus muscle is 10820 , and liver versus muscle is 9833 , respectively. These shared genes in each group were then used to calculate the fold change, which was defined as the ratio of RPKM values of brain versus liver, brain versus muscle, and liver versus muscle groups. In this study, the total fold change of $\geq 2$ was considered to classify the differentially expressed genes (DEGs). Based on this definition, there are 5228 DEGs between brain versus liver, 5283 between brain versus muscle, and 3840 between liver versus muscle. The list of DEGs in each group was further filtered by retaining only those genes that were annotated as a "glycoprotein" in the UniProt database. Therefore, 579, 501, and 442 differentially expressed glycogenes (DEGGs) for brain versus liver (BvL579), brain versus muscle (BvM501), and liver versus muscle (LvM442) groups were selected for the final analysis (Table 1(b)). The complete workflow of the analysis is shown in Figure 1.

3.2. Functional Annotation and Pathway Analysis. To classify biological processes that are enriched in the BvL579, BvM5, Annotation Cluster (FAC) tool available in the Database for Annotation, Visualization, and Integrated Discovery (DAVID) (http://david.abcc.ncifcrf.gov/home.jsp). The GO term "Biological Process" was used for annotations, and top 10 GO terms having statistically significant $P$ values from the resulting functional analysis for each group are listed in Table 2. From this table, it can be seen that the GO terms that are enriched in BvL579 glycogenes represent functions necessary for response to wounding, inflammatory response, and blood coagulation (Table 2(a)). The enriched GO terms in BvM501 include functions related to various metabolic (glycoprotein, glycosaminoglycan, sphingolipid, etc.) processes (Table 2(b)). Similarly, functions related to protein maturation, inflammatory response, and complement activation are enriched in LvM442 samples (Table 2(c)).

In addition to DAVID functional analysis, we also identified the biological pathways of BvL579, BvM501, and LvM442 DEGGs annotated in the present study. FASTA formatted amino acid sequences of DEGGs in these three sets were fed into the KAAS for prediction of distinct pathways. A total of 210 pathways were predicted for BvL579, whereas 193 and 198 pathways were predicted for BvM501 and LvM442, respectively. The top $10 \mathrm{KEGG}$ pathways for each of the three categories are shown in Table 3 and a complete list of all pathways is provided in Supplementary Table S1 (Supplementary Material is available online at http://dx.doi.org/10.1155/2014/837365). From these tables, it can be observed that the majority of the DEGGs were found to be associated with important biological processes, many being classified in signaling (e.g., PI3K-Akt and Rap1 signaling) pathways, cytokine-cytokine receptor interaction, or ECM-receptor interaction or being involved in adhesion related functions.

3.3. Interaction Network Construction and Identification of Hub Genes. DEGGs were mapped to the GeneMANIA to investigate how these genes interact with each other and 


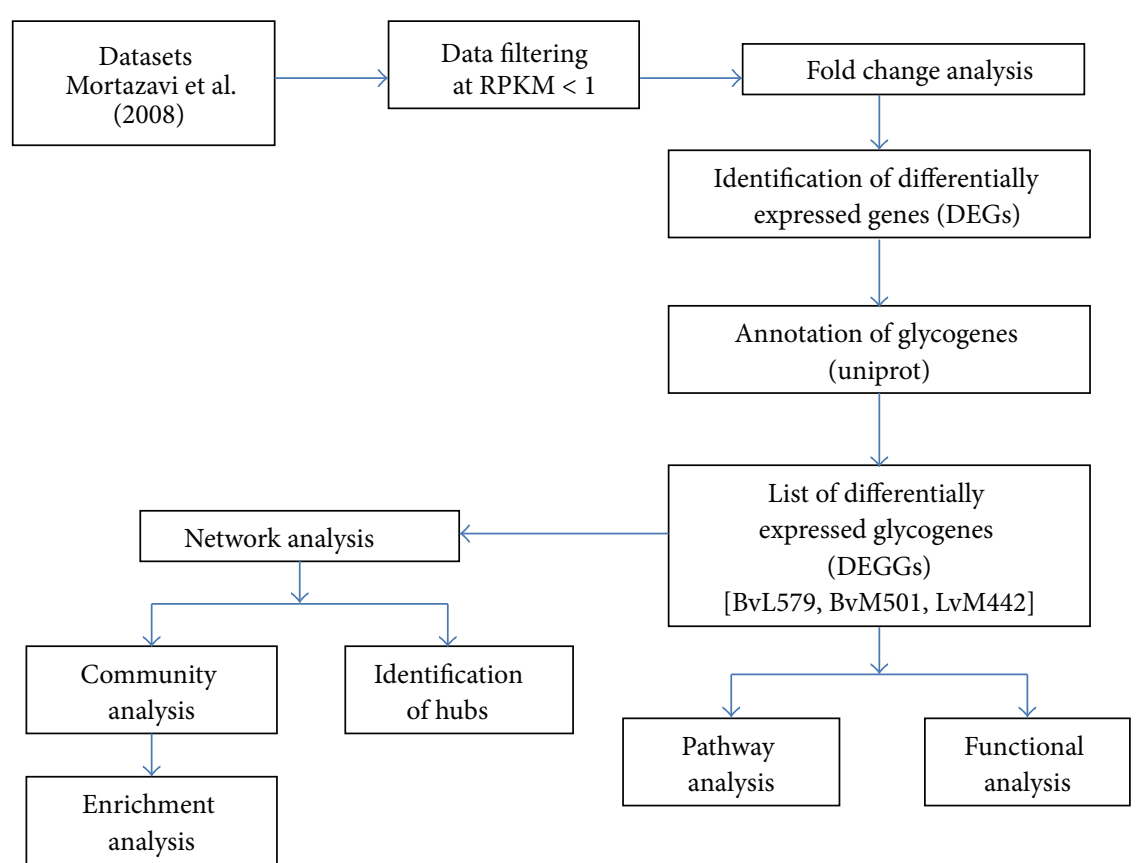

FIGURE 1: Flowchart depicting the overall methodology adopted in this study.

additional genes that are related to a set of query genes by using a very large set of functional interaction data [30]. The genes showing significant interactions with weights higher than 0.1 were selected only for the network analysis. By integrating these relationships, a network between DEGGs and additional related genes was constructed for all the three groups, namely, BvL579, BvM501, and LvM442 (Figure 2). A GeneMANIA network analysis for interactions among the glycogene products suggested enrichment of GO terms related to positive regulation of locomotion, cell motility, and migration for BvL579 (Table 4(a)), as well as LvM442 samples (Table 4(c)). On the contrary, the processes related to extracellular matrix were overrepresented in BvM501 samples (Table 4(b)).

The three interaction networks created from GeneMANIA were then exported to Cytoscape 2.8.2, a bioinformatics package for biological network visualization and data integration [31]. The initial network for BvL579 consists of 395 nodes and 1149 edges which were further filtered to 395 nodes and 1109 edges by removing duplicate edges (Figure 2(a)). In case of BvM501, the initial network consists of 352 nodes and 794 edges, whereas the filtered network has 352 nodes and 748 edges (Figure 2(b)). Similarly, the initial network for LvM442 network has 307 nodes and 695 edges, while the final network is represented by 307 nodes and 653 edges (Figure 2(c)). All the genes in the network are represented by circles and the interactions between them are represented as edges. Additionally, each query gene is shown in red whereas the additional related genes predicted by GeneMANIA are shown in cyan (Figure 2).

The node degree was then calculated for all the nodes in each network by using Network Analyzer plugin of
Cytoscape. The higher the node degree, the more important the gene was, and the gene was denoted as a hub gene. The genes with highest node degree include PEMT (BvL579) and IGf2 (BvM501), having node degree of 29 and 14, respectively. Both PEMT and IGF2 were predicted by GeneMANIA as related genes in their respective networks and do not represent the glycogenes. However, the genes with second highest node degrees of 23 and 13 are represented by glycogenes HPXN and NID2 in BvL579 and BvM501 networks (Figures 3(a) and 3(b)). Additionally, NID2 was also predicted by GeneMANIA as a related gene in BvM501 network because it was removed from the query list as it showed a fold change of less than 2 in our analysis. In LvM442 network, the top node degree genes are STAT6, and the glycogene FLT1 (Figure 3(c)), both having the node degree of 13.

3.4. Community Analysis and Functional Annotation of the Detected Modules. In order to identify the biologically related genes in the three networks, we employed Fast Greedy community-structure identification algorithm, implemented in the Cytoscape plugin GLay (http://brainarray.mbni.med.umich.edu/sugang/glay/) [29] to identify functional modules, 31 (Figure 4(a)), 40 (Figure 4(b)), and 31 (Figure 4(c)) clusters were detected for BvL579, BvM501, and LvM442 networks, respectively. However, only those communities were selected for enrichment analyses which have at least 10 nodes. Therefore, based on this criterion, 8, 9, and 8 communities for BvL579, BvM501, and LvM442 networks were finally analyzed for overrepresentation of GO terms. 
TABLE 2: Top 10 significantly enriched gene ontology (GO) terms detected by FAC in differentially expressed glycogenes between (a) BvL579, (b) BvM501, and (c) LvM442. Only those terms which reported a $P$ value of $\leq 0.05$ and count number $\geq 5$ genes were selected for the analysis.

(a) BvL579

\begin{tabular}{lcc}
\hline GO Term & Total number of genes & $P$ value \\
\hline GO:0009611 response to wounding & 53 & $2.98 E-23$ \\
GO:0002526 acute inflammatory response & 23 & $4.83 E-16$ \\
GO:0006954 inflammatory response & 35 & $1.43 E-15$ \\
GO:0051604 protein maturation & 23 & $2.51 E-14$ \\
GO:0007596 blood coagulation & 20 & $5.40 E-14$ \\
GO:0050817 coagulation & 20 & $5.40 E-14$ \\
GO:0007599 hemostasis & 20 & $7.17 E-14$ \\
GO:0006952 defense response & 45 & $7.57 E-13$ \\
GO:0050878 regulation of body fluid levels & 20 & $5.84 E-12$ \\
GO:0016485 protein processing & 20 & $7.21 E-12$ \\
\hline
\end{tabular}

(b) BvM501

\begin{tabular}{lcc}
\hline GO Term & Total number of genes & $P$ value \\
\hline GO:0009100 glycoprotein metabolic process & 30 & $1.76 E-18$ \\
GO:0009101 glycoprotein biosynthetic process & 23 & $8.1 E-14$ \\
GO:0070085 glycosylation & 17 & $6.09 E-10$ \\
GO:0006486 protein amino acid glycosylation & 17 & $6.09 E-10$ \\
GO:0043413 biopolymer glycosylation & 17 & $6.09 E-10$ \\
GO:0030203 glycosaminoglycan metabolic process & 11 & $3.1 E-08$ \\
GO:0006665 sphingolipid metabolic process & 12 & $2.79 E-07$ \\
GO:0006643 membrane lipid metabolic process & 12 & $3.9 E-07$ \\
GO:0006022 aminoglycan metabolic process & 11 & $0.45 E-07$ \\
GO:0051604 protein maturation & 14 & $6.1 E-07$ \\
\hline
\end{tabular}

(c) LvM442

\begin{tabular}{lcc}
\hline GO Term & Total number of genes & $P$ value \\
\hline GO:0051604 protein maturation & 20 & $3.17 E-13$ \\
GO:0016485 protein processing & 19 & $1.12 E-12$ \\
GO:0009100 glycoprotein metabolic process & 23 & $1.63 E-12$ \\
GO:0006954 inflammatory response & 27 & $6.69 E-12$ \\
GO:0009611 response to wounding & 33 & $1.07 E-11$ \\
GO:0002526 acute inflammatory response & 17 & $2.53 E-11$ \\
GO:0009101 glycoprotein biosynthetic process & 19 & $1.14 E-10$ \\
GO:0051605 protein maturation by peptide bond cleavage & 15 & $1.33 E-10$ \\
GO:0006956 complement activation & 11 & $4.25 E-09$ \\
GO:0002541 activation of plasma proteins involved in acute inflammatory response & 11 & $4.25 E-09$ \\
\hline
\end{tabular}

To biologically categorize these clusters, DAVID functional analysis tool was used to classify the genes in each module and observed the enrichment of GO term "Biological Process" in all the selected modules. The enrichment analysis for the modules for three networks is described as follows.

BvL579. The top 10 statistically significant enriched GO terms for DEGGs in all 8 clusters for BvL579 community analysis are summarized in Table 5(a). The most statistically significant GO terms that were enriched in cluster 1 were related to immune response, complement activation, and protein maturation. Clusters 2, 3, and 7 show enrichment for GO terms response to wounding, blood coagulation, and hemostasis. Clusters 2 and 3 also show overrepresentation of terms such as blood vessel development and vasculature development. The GO terms of cluster 4 were mostly related to hair cycle, development of epidermis, and ectoderm. The GO functions of cluster 5 were most related to localization, motility and migration of cells, and regulation of cell proliferation. Cluster 6 was significantly enriched with functions related to homeostasis, transport, and metabolism of cholesterol, and lipid as well as sterol. Cluster 8 showed overrepresentation of functional terms 
TABle 3: Top 10 KEGG pathways for DEGGs in each category. (a) BvL579, (b) BvM501, and (c) LvM442.

(a) BvL579

\begin{tabular}{lc}
\hline Pathway name & Number of mapped Genes \\
\hline 04151 PI3K-Akt signaling pathway & 34 \\
04610 Complement and coagulation cascades & 34 \\
04142 Lysosome & 31 \\
04060 Cytokine-cytokine receptor interaction & 23 \\
05200 Pathways in cancer & 21 \\
04015 Rap1 signaling pathway & 18 \\
04510 Focal adhesion & 18 \\
05205 Proteoglycans in cancer & 18 \\
04514 Cell adhesion molecules CAMs & 16 \\
04976 Bile secretion & 16 \\
\hline
\end{tabular}

(b) BvM501

\begin{tabular}{lc}
\hline Pathway name & Number of mapped Genes \\
\hline 04142 Lysosome & 29 \\
04151 PI3K-Akt signaling pathway & 24 \\
04060 Cytokine-cytokine receptor interaction & 19 \\
04514 Cell adhesion molecules CAMs & 19 \\
05200 Pathways in cancer & 17 \\
04141 Protein processing in endoplasmic reticulum & 15 \\
04510 Focal adhesion & 15 \\
05166 HTLV-I infection & 13 \\
04512 ECM-receptor interaction & 12 \\
04610 Complement and coagulation cascades & 12 \\
\hline
\end{tabular}

(c) LvM442

\begin{tabular}{lc}
\hline Pathway name & Number of mapped Genes \\
\hline 04142 Lysosome & 33 \\
04151 PI3K-Akt signaling pathway & 26 \\
04060 Cytokine-cytokine receptor interaction & 23 \\
04141 Protein processing in endoplasmic reticulum & 17 \\
05205 Proteoglycans in cancer & 17 \\
04610 Complement and coagulation cascades & 15 \\
04514 Cell adhesion molecules CAMs & 14 \\
04510 Focal adhesion & 14 \\
04512 ECM-receptor interaction & 13 \\
04145 Phagosome & 13 \\
\hline
\end{tabular}

related to the metabolism of glycosphingolipid, glycolipid, sphingolipid, and ganglioside.

BvM501. The top 10 statistically significant enriched GO terms for DEGGs in all 9 clusters for BvM501 community analysis are summarized in (Table 5(b)). Cluster 1 of this network shows overrepresentation of functions related to regulation of cell growth, protein maturation, and immune response. Cluster 2 is enriched in GO terms related to proteoglycan metabolic process as well as the development of blood vessel and vasculature. Cluster 3 is enriched in the functions related to the development of bone, cartilage, and skeletal system. The GO terms that are overrepresented in cluster 4 show enrichment for functions related to adhesion, such as biological adhesion, cell adhesion, cell-substrate adhesion, and cell-matrix adhesion. The processes related to localization, motility, and migration of cells are also overrepresented in cluster 4 . Cluster 5 showed overrepresentation of functional terms related to the metabolism of glycosphingolipid, glycolipid, sphingolipid, and ganglioside. Additionally, the functions related to the metabolism of lipid and oligosaccharides are also enriched in this group. In cluster 6, functions related to homeostasis, remodelling, and regulation of transport are overrepresented. Response to hypoxia and oxygen levels, apoptosis, and metabolic processes such as phospholipid and organophosphate metabolic process are enriched in cluster 7. Similar to cluster 4 , the GO terms that are overrepresented in Cluster 9 show enrichment 


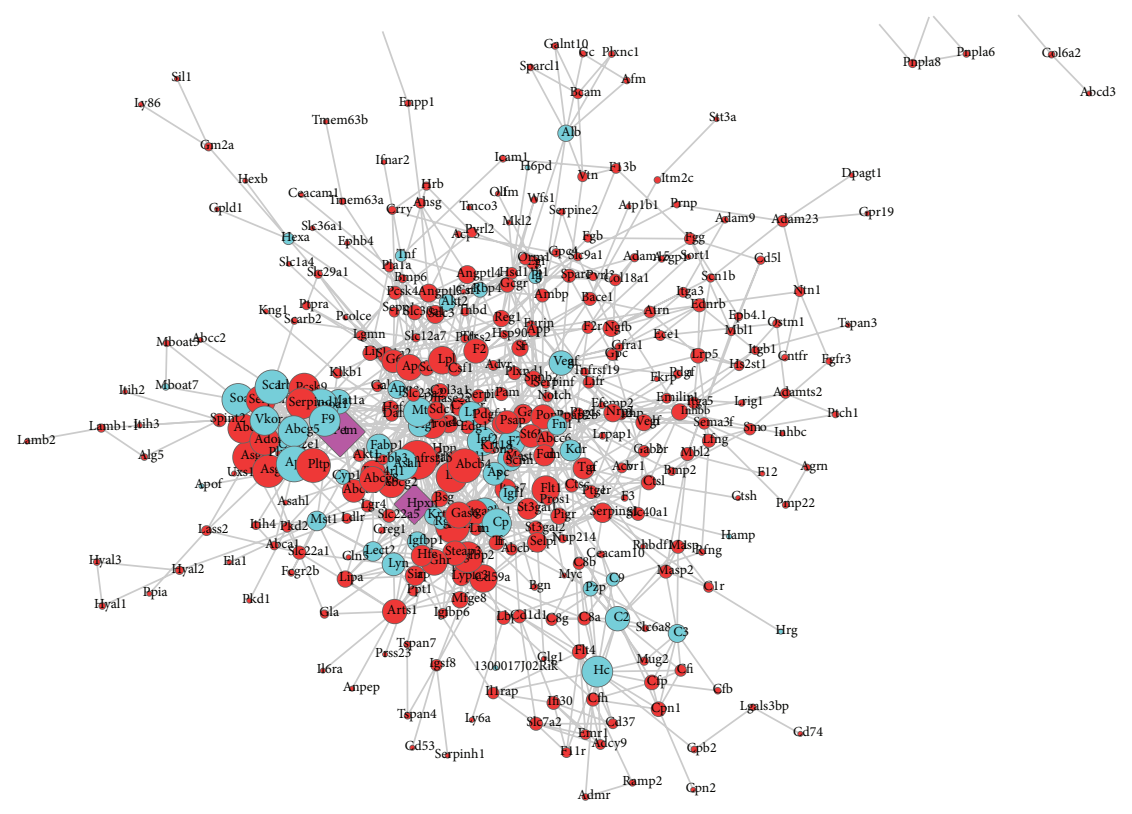

(a)

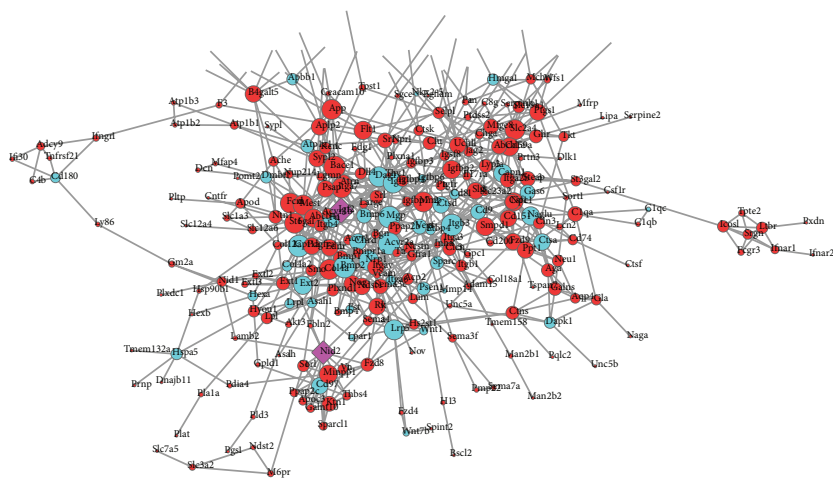

(b)

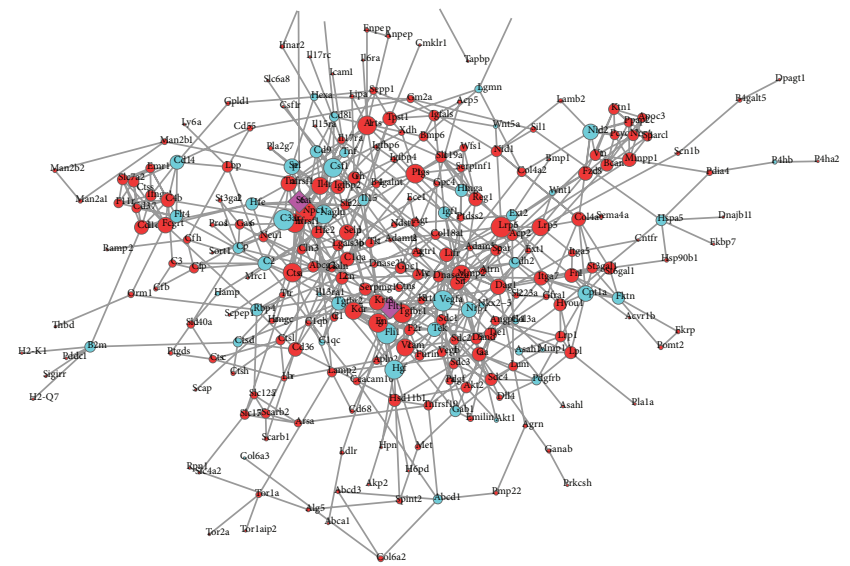

(c)

Figure 2: Interaction networks between DEGGs and additional related genes: (a) BvL579, (b) BvM501, and (c) LvM442. In each of these networks, red indicates a DEGG whereas the GeneMANIA predicted genes are shown in cyan. The top two hubs are shown as purple diamonds. The size of the nodes represents the node degree.

for functions related to biological adhesion. Protein folding and metal ion transport are the other important enriched functions present in this cluster. Cluster 8 did not yield the enrichment of any statistically significant GO category.

LvM442. Table 5(c) summarizes the top 10 statistically significant enriched GO terms for DEGGs in all 8 clusters for LvM442 community analysis. The GO terms related to development and morphogenesis of blood vessels, vasculature, and tube are enriched in cluster 1 . Cluster 2 shows enrichment of functions related to various types of responses, such as response to wounding, external stimulus, and defense as well as inflammatory response. Other enriched functional groups in this cluster are regulation of cell growth, proliferation, and cell activation. One of the overrepresented functional groups in cluster 3 shows enrichment for functions related to biological adhesion, lipid catabolic process, and 4-hydroxyproline metabolic process. Cluster 4 represents the enrichment of functions related to protein folding and bone remodeling and resorption. Cluster 5 shows overrepresentation of GO terms such as regulation of $\mathrm{T}$ cell mediated cytotoxicity or immunity, regulation of leukocyte mediated cytotoxicity, and regulation of cell killing. Clusters 6 and 7 are enriched in functions related to immune or inflammatory response and complement activation. Finally, cluster 8 exhibited overrepresentation of signaling pathways such as hepatocyte growth factor receptor signaling and mesenchymal-epithelial cell signaling, in addition to processes concerning MAP kinase activity. 
TABLE 4: 10 enriched gene ontology (GO) terms for DEGGs and additional related genes as reported by GeneMANIA for each category (a) BvL579, (b) BvM501, and (c) LvM442.

(a) BvL579

\begin{tabular}{lcr}
\hline GO id & Description & $q$-value \\
\hline GO:0040017 & positive regulation of locomotion & $1.83 E-23$ \\
GO:2000147 & positive regulation of cell motility & $1.83 E-23$ \\
GO:0030335 & positive regulation of cell migration & $3.40 E-23$ \\
GO:0051272 & positive regulation of cellular component movement & $3.40 E-23$ \\
GO:0050817 & coagulation & $1.94 E-20$ \\
GO:0010876 & lipid localization & $3.80 E-20$ \\
GO:0007596 & blood coagulation & $4.54 E-20$ \\
GO:0007599 & hemostasis & $7.56 E-20$ \\
GO:0009897 & external side of plasma membrane & $2.15 E-18$ \\
GO:0000323 & lytic vacuole & $1.05 E-17$ \\
\hline
\end{tabular}

(b) BvM501

\begin{tabular}{lcr}
\hline GO id & Description & $q$-value \\
\hline GO:0031012 & extracellular matrix & $2.43 E-23$ \\
GO:0005578 & proteinaceous extracellular matrix & $1.37 E-17$ \\
GO:0009100 & glycoprotein metabolic process & $1.99 E-16$ \\
GO:0000323 & lytic vacuole & $2.87 E-16$ \\
GO:0005764 & lysosome & $2.87 E-16$ \\
GO:0044420 & extracellular matrix part & $3.18 E-15$ \\
GO:0005604 & basement membrane & $1.20 E-14$ \\
GO:0005773 & vacuole & $4.56 E-14$ \\
GO:0030335 & positive regulation of cell migration & $5.17 E-12$ \\
GO:0005178 & integrin binding & $5.17 E-12$ \\
\hline
\end{tabular}

(c) LvM442

\begin{tabular}{lcr}
\hline GO id & Description & $q$-value \\
\hline GO:0000323 & lytic vacuole & $1.09 E-20$ \\
GO:0005764 & lysosome & $1.09 E-20$ \\
GO:0051272 & positive regulation of cellular component movement & $8.78 E-20$ \\
GO:0030335 & positive regulation of cell migration & $1.04 E-19$ \\
GO:2000147 & positive regulation of cell motility & $1.79 E-19$ \\
GO:0040017 & positive regulation of locomotion & $6.43 E-19$ \\
GO:0005773 & vacuole & $1.70 E-18$ \\
GO:0019838 & growth factor binding & $2.80 E-18$ \\
GO:0009100 & glycoprotein metabolic process & $2.11 E-15$ \\
GO:0031012 & extracellular matrix & $1.48 E-14$ \\
\hline
\end{tabular}

\section{Discussion}

The current study offers the first thorough insight into the glycogene analysis of brain, muscle, and liver tissues from mouse RNA-Seq data. Understanding the structure and function of these glycogenes is essential for studying the development of various tissues as well as their functional roles. Most of the serum glycoproteins originates from liver suggesting that liver diseases associated with aberrant glycosylation can be reflected by the changes in serum glycoproteins [32]. Recently, the significance of glycomics of central nervous system (CNS) to identify potential glycobiomarkers in neurological diseases [33] and alterations in brain glycoproteins resulting from the aging process was shown [34]. Recent studies in the glycomics field also offered insights into the biological significance of the glycome in the pathogenesis of diseases in humans [32]. Additionally, many studies have highlighted the significance of glycoconjugates during skeletal muscle development $[7,23,35]$.

With the evolution of bioinformatics during the past decades, molecular target discovery and targeted therapeutics have become a critical remedial treatment for diseases [36]. In this work, we screened DEGGs between brain, muscle, and liver tissues using RNA-Seq data and performed functional and pathway enrichment analysis using DAVID and KEGG tools. The most significant enriched GO terms 


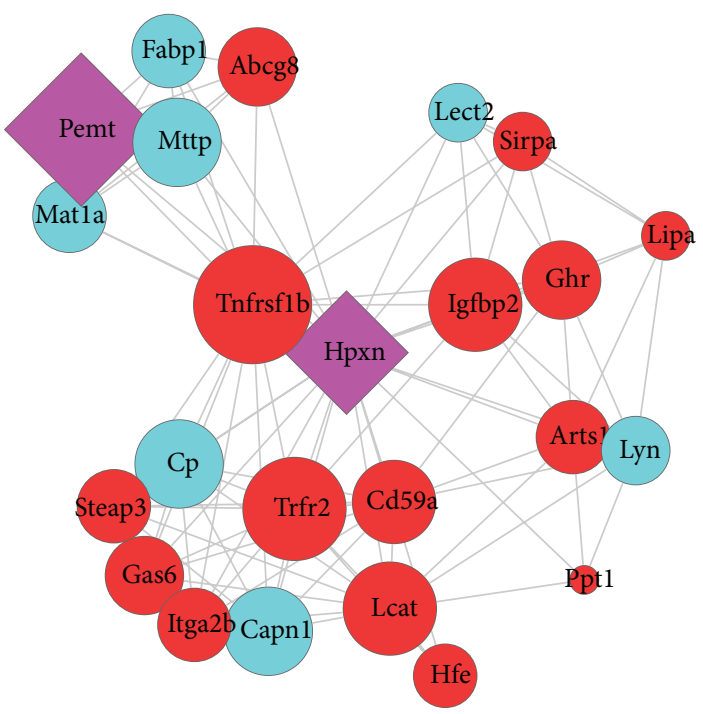

(a)

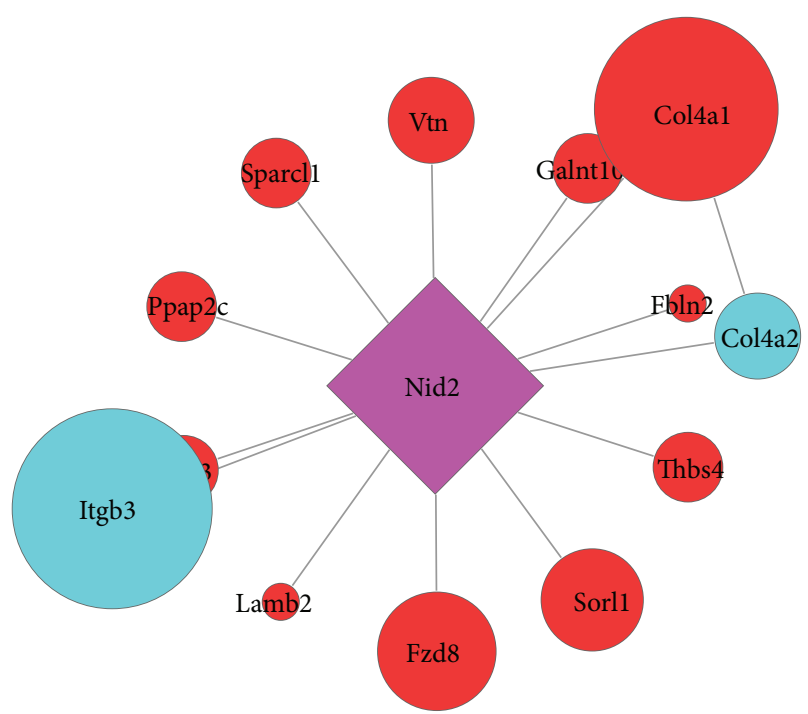

(b)

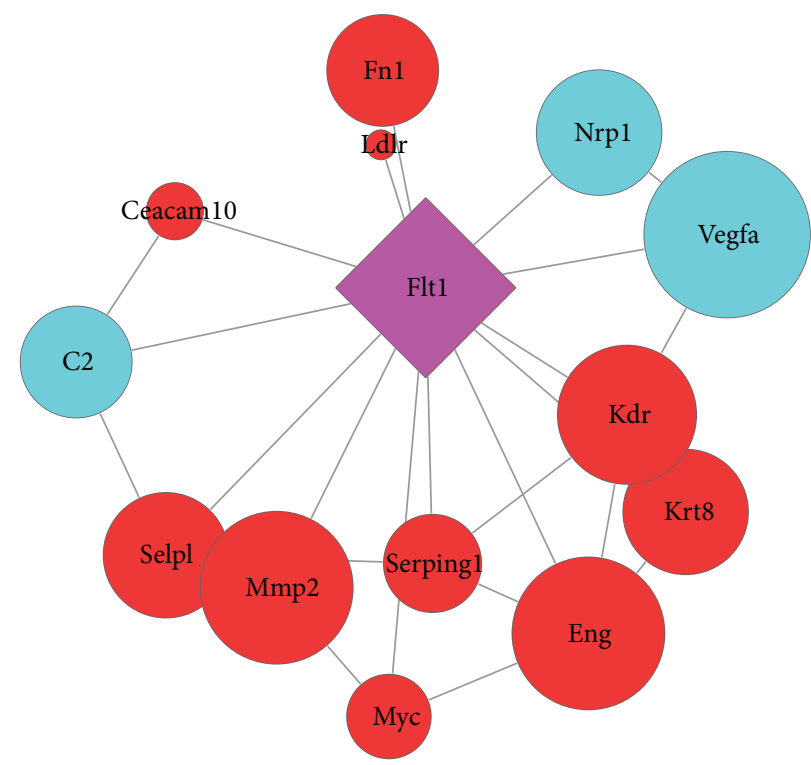

(c)

FIGURE 3: Top glycogenes as hubs and their first neighbors. (a) HPXN gene as hubs in BvL579 interaction network. (b) NID2 as hub gene in BvM501 interaction network. (c) FLT1 gene as hub in LvM442 interaction network.

for BvL579 are processes related to response to wounding or inflammatory response. These processes are represented by genes that play a prominent roles in innate immunity such as Mannan-binding lectin serine protease 1 (MASP1), Mannan-binding lectin serine protease 2 (MASP2), Mannosebinding protein $A(M B L 1)$, and Mannose-binding protein $C$ (MBL2) [37, 38]. BvM501 is enriched in functions related to glycoprotein metabolic processes and includes genes that code for various transferases such as $\mathrm{N}$-acetylglucosamine1-phosphotransferase subunit gamma (GNPTG) [39], Betagalactoside alpha-2,6-sialyltransferase 1 (ST6GAL1) [40], and Beta-1,3-galactosyltransferase 6 (B3GALT6) [41]. Similarly, the top statistically significant enriched processes in LvM442 are related to protein maturation or processing and are represented by genes such as Battenin (CLN3) and Methionine aminopeptidase 2 (METAP2). The CLN3 protein is involved in the late endosomal/lysosomal membrane transport [42], whereas METAP2 protein catalyzes the hydrolytic cleavage of the N-terminal methionine from newly synthesized polypeptides [43].

Additionally, three interaction networks of DEGGs were constructed for BvL579, BvM501, and LvM442 samples, and node degrees of genes were calculated. HPXN, NID2, and FLT1 were the glycogenes with the highest degree in BvL579, BvM501, and LvM442 networks, respectively. However, these genes represent the second highest node degree in their 

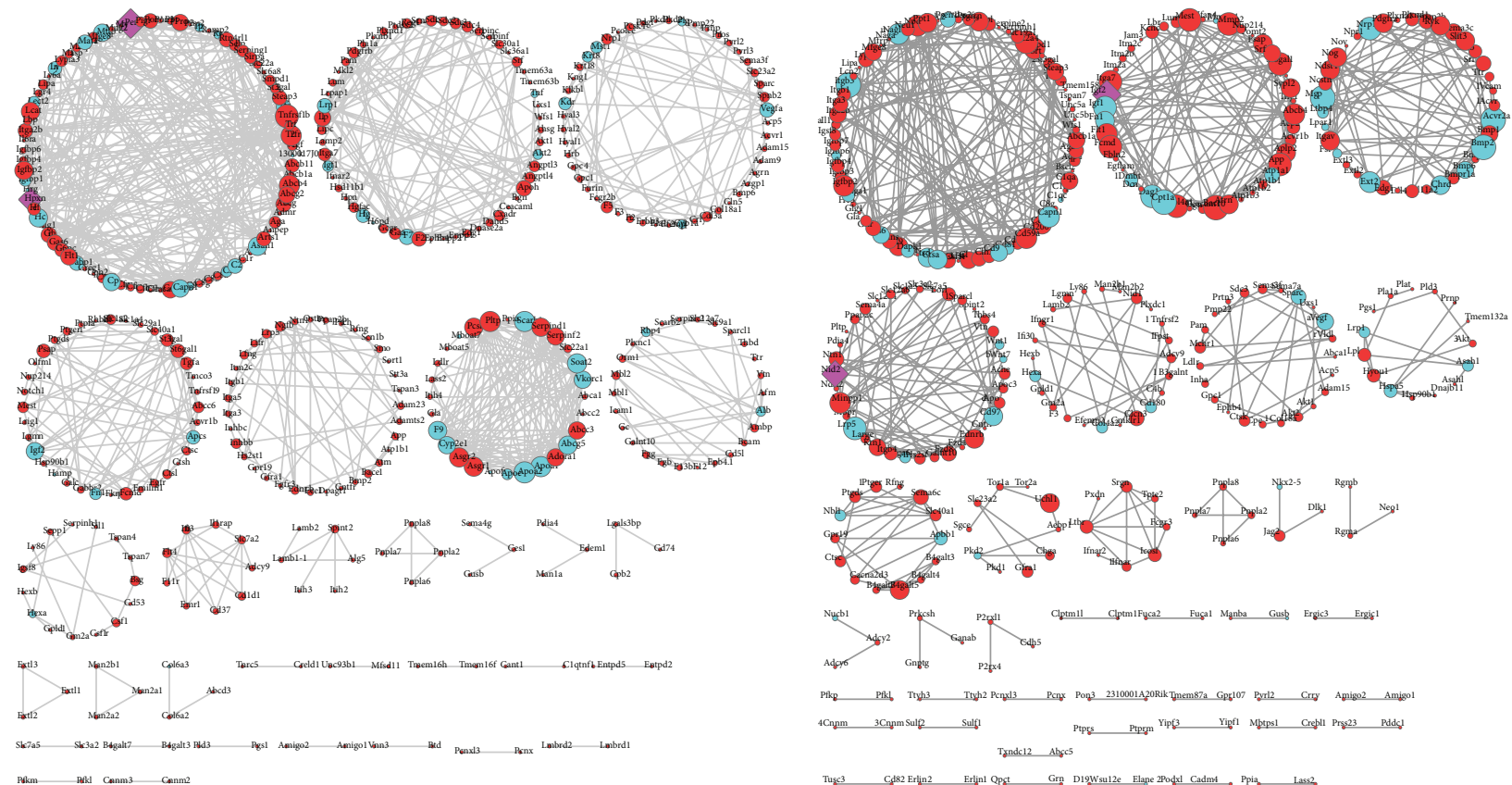

(a)

(b)
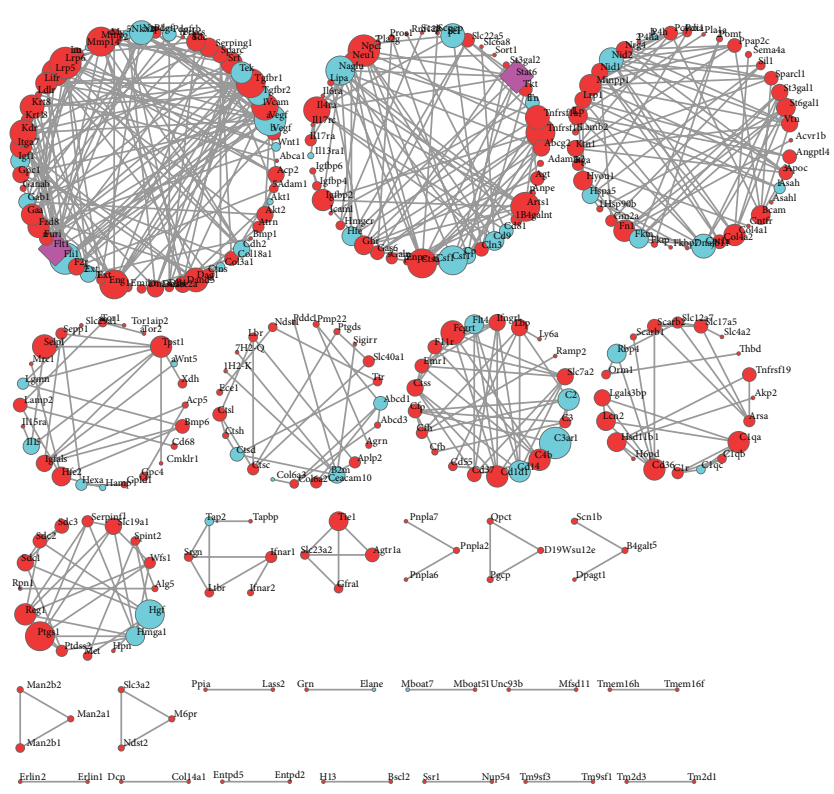

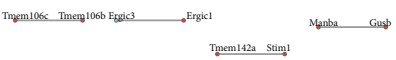

(c)

Figure 4: Communities generated by fast greedy (GLay) clustering algorithm are shown. Clusters for (a) BvL579 network, (b) BvM501 network, and (c) LvM442 network are shown. In each community, DEGG nodes are represented by red circles whereas the cyan nodes represent GeneMANIA predicted genes. Hub genes are shown as purple diamonds.

respective networks as genes (PEMT, IGF2, and STAT6) with top node degree that do not code for glycoproteins.

HPXN ( $60 \mathrm{kDa}$ glycoprotein) is mainly synthesized by liver cells [44], secreted to the plasma where it binds free heme or hemin and inhibits its role in free radical reactions [45]. HPXN is also reported to be expressed by the cells of immune systems, ganglionic and photoreceptor cells of the retina, cells of the peripheral nervous system, and the mesangial cells of kidney [46-48]. Recently, it was shown that HPX protein is also present in various regions of mouse brain [49]. NID2 is homologous to another member of the nidogen family, NID1, and both are found in all basement membranes (BMs) [50]. Both nidogens have a similar distribution in various organs during development; however, in 
TABLE 5: Enrichment of GO terms for BvL579, BvM501, and LvM442 cluster analysis.

(a) BvL579

\begin{tabular}{|c|c|c|}
\hline Cluster number & Term & $P$ value \\
\hline \multirow{10}{*}{1} & GO:0002541 activation of plasma proteins involved in acute inflammatory response & $5.02 E-22$ \\
\hline & GO:0006956 complement activation & $5.02 E-22$ \\
\hline & GO:0002526 acute inflammatory response & $2.26 E-20$ \\
\hline & GO:0006959 humoral immune response & $2.25 E-19$ \\
\hline & GO:0051604 protein maturation & $3.43 E-19$ \\
\hline & GO:0051605 protein maturation by peptide bond cleavage & $3.19 E-18$ \\
\hline & GO:0002253 activation of immune response & $3.20 E-18$ \\
\hline & GO:0016485 protein processing & $6.27 E-18$ \\
\hline & GO:0006957 complement activation, alternative pathway & $8.91 E-18$ \\
\hline & GO:0006954 inflammatory response & $1.44 E-17$ \\
\hline \multirow{10}{*}{2} & GO:0051094 positive regulation of developmental process & $5.33 E-05$ \\
\hline & GO:0042592 homeostatic process & $4.25 E-04$ \\
\hline & GO:0009611 response to wounding & $7.29 E-04$ \\
\hline & GO:0001568 blood vessel development & $9.88 E-04$ \\
\hline & GO:0001944 vasculature development & 0.001101764 \\
\hline & GO:0050817 coagulation & 0.001394123 \\
\hline & GO:0007596 blood coagulation & 0.001394123 \\
\hline & GO:0007599 hemostasis & 0.001452434 \\
\hline & GO:0050878 regulation of body fluid levels & 0.002774109 \\
\hline & GO:0007169 transmembrane receptor protein tyrosine kinase signaling pathway & 0.003100357 \\
\hline \multirow{10}{*}{3} & GO:0050817 coagulation & $2.74 E-11$ \\
\hline & GO:0007596 blood coagulation & $2.74 E-11$ \\
\hline & GO:0007599 hemostasis & $3.08 E-11$ \\
\hline & GO:0050878 regulation of body fluid levels & $1.97 E-10$ \\
\hline & GO:0042060 wound healing & $1.26 E-09$ \\
\hline & GO:0009611 response to wounding & $5.75 E-08$ \\
\hline & GO:0007167 enzyme linked receptor protein signaling pathway & $9.33 E-08$ \\
\hline & GO:0001568 blood vessel development & $5.52 E-07$ \\
\hline & GO:0001944 vasculature development & $6.64 E-07$ \\
\hline & GO:0001525 angiogenesis & $4.71 E-05$ \\
\hline \multirow{10}{*}{4} & GO:0022404 molting cycle process & $2.16 E-04$ \\
\hline & GO:0022405 hair cycle process & $2.16 E-04$ \\
\hline & GO:0001942 hair follicle development & $2.16 E-04$ \\
\hline & GO:0042633 hair cycle & $2.42 E-04$ \\
\hline & GO:0042303 molting cycle & $2.42 E-04$ \\
\hline & GO:0008544 epidermis development & 0.003102493 \\
\hline & GO:0007398 ectoderm development & 0.00369522 \\
\hline & GO:0050678 regulation of epithelial cell proliferation & 0.009595347 \\
\hline & GO:0048732 gland development & 0.01092817 \\
\hline & GO:0007173 epidermal growth factor receptor signaling pathway & 0.025611634 \\
\hline \multirow{10}{*}{5} & GO:0007166 cell surface receptor linked signal transduction & $1.34 E-05$ \\
\hline & GO:0016477 cell migration & $2.69 E-05$ \\
\hline & GO:0042127 regulation of cell proliferation & $3.44 E-05$ \\
\hline & GO:0051674 localization of cell & $4.88 E-05$ \\
\hline & GO:0048870 cell motility & $4.88 E-05$ \\
\hline & GO:0006928 cell motion & $6.35 E-05$ \\
\hline & GO:0008284 positive regulation of cell proliferation & $2.52 E-04$ \\
\hline & GO:0007229 integrin-mediated signaling pathway & $4.87 E-04$ \\
\hline & GO:0040007 growth & $6.74 E-04$ \\
\hline & GO:0007507 heart development & 0.001229375 \\
\hline
\end{tabular}


(a) Continued.

\begin{tabular}{|c|c|c|}
\hline Cluster number & Term & $P$ value \\
\hline \multirow{10}{*}{6} & GO:0055088 lipid homeostasis & $2.16 E-16$ \\
\hline & GO:0042632 cholesterol homeostasis & $2.64 E-15$ \\
\hline & GO:0055092 sterol homeostasis & $2.64 E-15$ \\
\hline & GO:0030301 cholesterol transport & $3.97 E-13$ \\
\hline & GO:0015918 sterol transport & $3.97 E-13$ \\
\hline & GO:0033344 cholesterol efflux & $7.23 E-13$ \\
\hline & GO:0006869 lipid transport & $3.89 E-12$ \\
\hline & GO:0008203 cholesterol metabolic process & $4.74 E-12$ \\
\hline & GO:0010876 lipid localization & $8.01 E-12$ \\
\hline & GO:0016125 sterol metabolic process & $1.02 E-11$ \\
\hline \multirow{10}{*}{7} & GO:0009611 response to wounding & $3.88 E-07$ \\
\hline & GO:0007596 blood coagulation & $2.94 E-06$ \\
\hline & GO:0050817 coagulation & $2.94 E-06$ \\
\hline & GO:0007599 hemostasis & $3.11 E-06$ \\
\hline & GO:0050878 regulation of body fluid levels & $7.69 E-06$ \\
\hline & GO:0042060 wound healing & $1.91 E-05$ \\
\hline & GO:0051818 disruption of cells of other organism during symbiotic interaction & $3.08 E-05$ \\
\hline & GO:0051851 modification by host of symbiont morphology or physiology & $3.08 E-05$ \\
\hline & GO:0051883 killing of cells in other organism during symbiotic interaction & $3.08 E-05$ \\
\hline & GO:0031640 killing of cells of another organism & $4.30 E-05$ \\
\hline \multirow{10}{*}{8} & GO:0006689 ganglioside catabolic process & $5.11 E-06$ \\
\hline & GO:0046479 glycosphingolipid catabolic process & $1.79 E-05$ \\
\hline & GO:0019377 glycolipid catabolic process & $1.79 E-05$ \\
\hline & GO:0001573 ganglioside metabolic process & $2.38 E-05$ \\
\hline & GO:0046466 membrane lipid catabolic process & $1.15 E-04$ \\
\hline & GO:0030149 sphingolipid catabolic process & $1.15 E-04$ \\
\hline & GO:0019915 lipid storage & $1.29 E-04$ \\
\hline & GO:0006687 glycosphingolipid metabolic process & $2.73 E-04$ \\
\hline & GO:0006664 glycolipid metabolic process & $3.90 E-04$ \\
\hline & GO:0050885 neuromuscular process controlling balance & $4.98 E-04$ \\
\hline
\end{tabular}

(b) BvM501

\begin{tabular}{llr}
\hline Cluster number & Term & $P$ value \\
\hline \multicolumn{1}{c}{ GO:0040008 regulation of growth } & $3.73 E-08$ \\
GO:0051604 protein maturation & $7.27 E-08$ \\
GO:0001558 regulation of cell growth & $1.29 E-06$ \\
GO:0016485 protein processing & $2.32 E-05$ \\
GO:0016044 membrane organization & $7.38 E-05$ \\
GO:0016064 immunoglobulin mediated immune response & $9.61 E-05$ \\
GO:0051605 protein maturation by peptide bond cleavage & $1.09 E-04$ \\
GO:0019724 B cell mediated immunity & $1.09 E-04$ \\
GO:0002684 positive regulation of immune system process & $1.29 E-04$ \\
GO:0006958 complement activation, classical pathway & $2.00 E-04$ \\
\hline & GO:0009100 glycoprotein metabolic process & $2.16 E-06$ \\
GO:0050654 chondroitin sulfate proteoglycan metabolic process & $9.34 E-06$ \\
GO:0006029 proteoglycan metabolic process & $8.82 E-05$ \\
GO:0048754 branching morphogenesis of a tube & $1.02 E-04$ \\
GO:0019800 peptide cross-linking via chondroitin 4 -sulfate glycosaminoglycan & $1.89 E-04$ \\
GO:0001763 morphogenesis of a branching structure & $3.20 E-04$ \\
GO:0001568 blood vessel development & $4.29 E-04$ \\
GO:0001944 vasculature development & $4.79 E-04$ \\
GO:0030204 chondroitin sulfate metabolic process & $6.09 E-04$ \\
GO:0006813 potassium ion transport & $8.13 E-04$ \\
\hline
\end{tabular}


(b) Continued.

\begin{tabular}{|c|c|c|}
\hline Cluster number & Term & $P$ value \\
\hline \multirow{10}{*}{3} & GO:0001501 skeletal system development & $1.07 E-16$ \\
\hline & GO:0030509 BMP signaling pathway & $6.34 E-14$ \\
\hline & GO:0001503 ossification & $4.02 E-12$ \\
\hline & GO:0060348 bone development & $7.43 E-12$ \\
\hline & GO:0030500 regulation of bone mineralization & $6.08 E-11$ \\
\hline & GO:0007178 transmembrane receptor protein serine/threonine kinase signaling pathway & $7.75 E-11$ \\
\hline & GO:0070167 regulation of biomineral formation & $9.25 E-11$ \\
\hline & GO:0007167 enzyme linked receptor protein signaling pathway & $1.84 E-10$ \\
\hline & GO:0007369 gastrulation & $2.22 E-10$ \\
\hline & GO:0051216 cartilage development & $3.31 E-10$ \\
\hline \multirow{10}{*}{4} & GO:0006928 cell motion & $9.88 E-05$ \\
\hline & GO:0016055 Wnt receptor signaling pathway & $2.59 E-04$ \\
\hline & GO:0016477 cell migration & $4.37 E-04$ \\
\hline & GO:0048870 cell motility & $7.09 E-04$ \\
\hline & GO:0051674 localization of cell & $7.09 E-04$ \\
\hline & GO:0007160 cell-matrix adhesion & 0.001189285 \\
\hline & GO:0009100 glycoprotein metabolic process & 0.001250564 \\
\hline & GO:0031589 cell-substrate adhesion & 0.001570006 \\
\hline & GO:0007155 cell adhesion & 0.005368157 \\
\hline & GO:0022610 biological adhesion & 0.005405426 \\
\hline \multirow{10}{*}{5} & GO:0006689 ganglioside catabolic process & $1.66 E-05$ \\
\hline & GO:0019377 glycolipid catabolic process & $5.78 E-05$ \\
\hline & GO:0046479 glycosphingolipid catabolic process & $5.78 E-05$ \\
\hline & GO:0001573 ganglioside metabolic process & $7.69 E-05$ \\
\hline & GO:0009611 response to wounding & $2.01 E-04$ \\
\hline & GO:0046466 membrane lipid catabolic process & $3.70 E-04$ \\
\hline & GO:0030149 sphingolipid catabolic process & $3.70 E-04$ \\
\hline & GO:0019915 lipid storage & $4.16 E-04$ \\
\hline & GO:0006687 glycosphingolipid metabolic process & $8.77 E-04$ \\
\hline & GO:0009311 oligosaccharide metabolic process & 0.001247784 \\
\hline \multirow{10}{*}{6} & GO:0042592 homeostatic process & $6.03 E-06$ \\
\hline & GO:0043270 positive regulation of ion transport & 0.001725638 \\
\hline & GO:0051270 regulation of cell motion & 0.002725255 \\
\hline & GO:0048771 tissue remodeling & 0.003048492 \\
\hline & GO:0001894 tissue homeostasis & 0.003842174 \\
\hline & GO:0051050 positive regulation of transport & 0.004095371 \\
\hline & GO:0007169 transmembrane receptor protein tyrosine kinase signaling pathway & 0.004147057 \\
\hline & GO:0032869 cellular response to insulin stimulus & 0.004461779 \\
\hline & GO:0042127 regulation of cell proliferation & 0.004903945 \\
\hline & GO:0010765 positive regulation of sodium ion transport & 0.005901212 \\
\hline \multirow{9}{*}{7} & GO:0001666 response to hypoxia & 0.006023043 \\
\hline & GO:0070482 response to oxygen levels & 0.006648281 \\
\hline & GO:0016042 lipid catabolic process & 0.009864913 \\
\hline & GO:0006644 phospholipid metabolic process & 0.011805904 \\
\hline & GO:0019637 organophosphate metabolic process & 0.013020542 \\
\hline & GO:0006916 anti-apoptosis & 0.013774773 \\
\hline & GO:0043066 negative regulation of apoptosis & 0.037899056 \\
\hline & GO:0043069 negative regulation of programmed cell death & 0.038883095 \\
\hline & GO:0060548 negative regulation of cell death & 0.03908111 \\
\hline
\end{tabular}


(b) Continued.

\begin{tabular}{llr}
\hline Cluster number & Term & $P$ value \\
\hline 8 & NA & NA \\
\hline & GO:0007160 cell-matrix adhesion & 0.001495186 \\
GO:0031589 cell-substrate adhesion & 0.001809131 \\
GO:0051085 chaperone mediated protein folding requiring cofactor & 0.006635187 \\
GO:0051084 “de novo" posttranslational protein folding & 0.008618097 \\
GO:0007155 cell adhesion & 0.00916406 \\
9 & GO:0022610 biological adhesion & 0.009200307 \\
GO:0050982 detection of mechanical stimulus & 0.010597488 \\
GO:0006458 “de novo" protein folding & 0.010597488 \\
GO:0007259 JAK-STAT cascade & 0.025656487 \\
GO:0030001 metal ion transport & 0.036163834 \\
GO:0030001 metal ion transport & 0.036163834 \\
\hline
\end{tabular}

(c) LvM442

\begin{tabular}{|c|c|c|}
\hline Cluster number & Term & $P$ value \\
\hline \multirow{10}{*}{1} & GO:0001568 blood vessel development & $1.06 E-18$ \\
\hline & GO:0001944 vasculature development & $1.64 E-18$ \\
\hline & GO:0048514 blood vessel morphogenesis & $8.45 E-16$ \\
\hline & GO:0001525 angiogenesis & $3.48 E-12$ \\
\hline & GO:0048754 branching morphogenesis of a tube & $9.17 E-11$ \\
\hline & GO:0007167 enzyme linked receptor protein signaling pathway & $5.50 E-10$ \\
\hline & GO:0035239 tube morphogenesis & $1.14 E-09$ \\
\hline & GO:0001763 morphogenesis of a branching structure & $1.32 E-09$ \\
\hline & GO:0035295 tube development & $5.53 E-09$ \\
\hline & GO:0001569 patterning of blood vessels & $3.29 E-08$ \\
\hline \multirow{10}{*}{2} & GO:0040008 regulation of growth & $4.27 E-07$ \\
\hline & GO:0009611 response to wounding & $4.18 E-06$ \\
\hline & GO:0006954 inflammatory response & $3.42 E-05$ \\
\hline & GO:0032101 regulation of response to external stimulus & $1.89 E-04$ \\
\hline & GO:0048585 negative regulation of response to stimulus & $7.82 E-04$ \\
\hline & GO:0006952 defense response & 0.001399518 \\
\hline & GO:0001558 regulation of cell growth & 0.002134607 \\
\hline & GO:0050867 positive regulation of cell activation & 0.002784166 \\
\hline & GO:0032102 negative regulation of response to external stimulus & 0.004518678 \\
\hline & GO:0008283 cell proliferation & 0.004842417 \\
\hline \multirow{10}{*}{3} & GO:0007160 cell-matrix adhesion & $7.84 E-05$ \\
\hline & GO:0031589 cell-substrate adhesion & $1.14 E-04$ \\
\hline & GO:0044242 cellular lipid catabolic process & $9.80 E-04$ \\
\hline & GO:0016042 lipid catabolic process & $9.93 E-04$ \\
\hline & GO:0051346 negative regulation of hydrolase activity & 0.006807864 \\
\hline & GO:0008218 bioluminescence & 0.007742186 \\
\hline & GO:0018401 peptidyl-proline hydroxylation to 4-hydroxy-L-proline & 0.007742186 \\
\hline & GO:0019471 4-hydroxyproline metabolic process & 0.007742186 \\
\hline & GO:0009101 glycoprotein biosynthetic process & 0.007780584 \\
\hline & GO:0007155 cell adhesion & 0.00843311 \\
\hline \multirow{8}{*}{4} & GO:0001501 skeletal system development & $7.15 E-04$ \\
\hline & GO:0006955 immune response & 0.011579 \\
\hline & GO:0051085 chaperone mediated protein folding requiring cofactor & 0.013230724 \\
\hline & GO:0051084 "de novo" posttranslational protein folding & 0.01716759 \\
\hline & GO:0045453 bone resorption & 0.019783921 \\
\hline & GO:0006458 “de novo" protein folding & 0.021089619 \\
\hline & GO:0046849 bone remodeling & 0.027593503 \\
\hline & GO:0006952 defense response & 0.045862312 \\
\hline
\end{tabular}


(c) Continued.

\begin{tabular}{|c|c|c|}
\hline Cluster number & Term & $P$ value \\
\hline \multirow{10}{*}{5} & GO:0001916 positive regulation of T cell mediated cytotoxicity & $4.08 E-05$ \\
\hline & GO:0001914 regulation of T cell mediated cytotoxicity & $6.22 E-05$ \\
\hline & GO:0002711 positive regulation of $\mathrm{T}$ cell mediated immunity & $8.81 E-05$ \\
\hline & GO:0002474 antigen processing and presentation of peptide antigen via MHC class I & $1.53 E-04$ \\
\hline & GO:0002709 regulation of T cell mediated immunity & $1.72 E-04$ \\
\hline & GO:0031343 positive regulation of cell killing & $2.84 E-04$ \\
\hline & GO:0001912 positive regulation of leukocyte mediated cytotoxicity & $2.84 E-04$ \\
\hline & GO:0001910 regulation of leukocyte mediated cytotoxicity & $4.86 E-04$ \\
\hline & GO:0031341 regulation of cell killing & $4.86 E-04$ \\
\hline & GO:0002821 positive regulation of adaptive immune response & $6.25 E-04$ \\
\hline \multirow{10}{*}{6} & GO:0002526 acute inflammatory response & $4.67 E-14$ \\
\hline & GO:0045087 innate immune response & $4.65 E-13$ \\
\hline & GO:0002684 positive regulation of immune system process & $1.53 E-12$ \\
\hline & GO:0050778 positive regulation of immune response & $3.29 E-12$ \\
\hline & GO:0006954 inflammatory response & $3.41 E-12$ \\
\hline & GO:0002541 activation of plasma proteins involved in acute inflammatory response & $4.05 E-12$ \\
\hline & GO:0006956 complement activation & $4.05 E-12$ \\
\hline & GO:0048584 positive regulation of response to stimulus & $4.13 E-11$ \\
\hline & GO:0006952 defense response & $4.75 E-11$ \\
\hline & GO:0006959 humoral immune response & $5.29 E-11$ \\
\hline \multirow{10}{*}{7} & GO:0009611 response to wounding & $2.97 E-06$ \\
\hline & GO:0006958 complement activation, classical pathway & $7.07 E-06$ \\
\hline & GO:0002526 acute inflammatory response & $7.33 E-06$ \\
\hline & GO:0002455 humoral immune response mediated by circulating immunoglobulin & $8.69 E-06$ \\
\hline & GO:0006956 complement activation & $2.20 E-05$ \\
\hline & GO:0002541 activation of plasma proteins involved in acute inflammatory response & $2.36 E-05$ \\
\hline & GO:0016064 immunoglobulin mediated immune response & $4.70 E-05$ \\
\hline & GO:0019724 B cell mediated immunity & $5.25 E-05$ \\
\hline & GO:0002449 lymphocyte mediated immunity & $1.02 E-04$ \\
\hline & GO:0002250 adaptive immune response & $1.36 E-04$ \\
\hline \multirow{9}{*}{8} & GO:0051450 myoblast proliferation & 0.002206368 \\
\hline & GO:0048012 hepatocyte growth factor receptor signaling pathway & 0.002206368 \\
\hline & $\begin{array}{l}\text { GO:0060665 regulation of branching involved in salivary gland morphogenesis by } \\
\text { mesenchymal-epithelial signaling }\end{array}$ & 0.003674845 \\
\hline & GO:0060638 mesenchymal-epithelial cell signaling & 0.004408354 \\
\hline & GO:0060693 regulation of branching involved in salivary gland morphogenesis & 0.006605966 \\
\hline & GO:0060688 regulation of morphogenesis of a branching structure & 0.018252999 \\
\hline & GO:0001889 liver development & 0.031208893 \\
\hline & GO:0000187 activation of MAPK activity & 0.036205594 \\
\hline & GO:0043406 positive regulation of MAP kinase activity & 0.042595839 \\
\hline
\end{tabular}

adult tissues nidogen-2 distribution becomes more confined [51-53]. Additionally, these nidogens show a broad range of interacting partners including other BM proteins such as laminin, collagen IV, and perlecan [51, 53-55]. They are involved in various functions including the regulation of cell attachment [56], neutrophil chemotaxis [57], trophoblast outgrowth [58], angiogenesis [59], osteoblast, and myogenic differentiation [60, 61]. Vascular endothelial growth factors (VEGFs) constitute a family of six polypeptides (VEGF$\mathrm{A},-\mathrm{B},-\mathrm{C},-\mathrm{D},-\mathrm{E}$, and $\mathrm{PlGF})$ that regulate blood and lymphatic vessel development [62]. VEGF signaling occurs by binding to various cellular receptors such as VEGFR1 (FLT1), VEGFR2 (FLK1), and VEGFR3 (FLT4) $[63,64]$ and neuropilin-1 [65] and -2 (NRP1 and NRP2) [66] and heparan sulfate proteoglycans (HSPG) [67]. FLT1 and FLK1 are closely related receptor tyrosine kinases and both share common and specific ligands [68]. FLT1 has weaker kinase activity than FLK1 [68]; however, FLT1 is essential for normal development and angiogenesis as reported in previous FLT1 null mutant mice studies [69-71].

Recognizing the structure and function of biological networks is indispensable for the investigation of biological processes. In this work, we identified 8,9 , and 8 functional modules or communities for BvL579, BvM501, and LvM442 networks using fast greedy algorithm implemented as GLAY [29], plugin for cytoscape. Furthermore, the function of 
each module in all the three networks was explored by using functional annotation tool DAVID. Our analysis shows that the modules that are common in BvL579 and LvM442 networks show enrichment for processes related to immune and inflammatory response and response to wounding. Liver is known to play an important role in innate immunity, an important primary line of defense against infection [72]. Additionally, Kupffer cells in the liver are one of the earliest to be affected by bacterial or sterile insults and add to the inflammatory response [73]. This sterile inflammation can be responsible for liver injury; it may also play a role in liver repair [72]. In case of the modules of BvM501 and LvM442 networks, the enrichment of processes related to cell adhesion, proliferation, and regulation of cell growth is observed. The expression of myogenic regulatory factors (MRFs) describes the different stages of skeletal muscle development that includes myoblast proliferation, cell-cycle exit, cell fusion, and the maturation of myotubes to form myofibers [23]. Cell adhesion molecules such as cadherins are glycoproteins that mediate homotypic cell-cell adhesion through their extracellular domain [74], and this cadherindependent adhesion is necessary for diverse types of cellular functions [75]. Finally, glycosphingolipid or glycolipid metabolic processes show overrepresentation in modules of BvL579 and BvM501 networks. Glycosphingolipids are universally expressed in all vertebrate cells as well as body fluids, but they are richer in the nervous system [76]. Previous studies have established the role of glycosphingolipids in the development of the brain among various species [77, 78].

\section{Conclusion}

Overall, in this work we have used a systems biology approach to identify the DEGGs, their functional enrichment, and identified potential hub genes by constructing three glycogene interaction networks. We also identified functional modules in these networks and indicate the significance of immune system related processes in liver, and glycosphingolipid metabolic processes in the development of brain. Similarly, we observe that the processes such as cell proliferation, adhesion, and growth are important for muscle development. The findings deduced from the current work are in consistence with the previous studies. Experimental validation will be required to confirm the predictions made in this study that will establish the role of predicted hubs as well as enriched functional processes in these tissues.

\section{Conflict of Interests}

The authors declare that there is no conflict of interests regarding the publication of this paper.

\section{Acknowledgments}

Authors would like to acknowledge the support from the Biomedical Informatics Center of ICMR, PGIMER (Chandigarh, India); School of Chemistry and Biochemistry, Thapar University, (Patiala, India); and School of Biotechnology,
Yeungnam University (Gyeongsan, South Korea) for providing lab facilities to conduct this research.

\section{References}

[1] J. Salzman, H. Jiang, and W. H. Wong, "Statistical modeling of RNA-Seq data," Statistical Science, vol. 26, no. 1, pp. 62-83, 2011.

[2] F. Mantelli, L. Schaffer, R. Dana, S. R. Head, and P. Argüeso, "Glycogene expression in conjunctiva of patients with dry eye: downregulation of Notch signaling," PLOS ONE, vol. 50, no. 6, pp. 2666-2672, 2010.

[3] A. Ishii, S. Hitoshi, I. Fujimoto et al., "Developmental changes in the expression of glycogenes and the content of $N$-glycans in the mouse cerebral cortex," Glycobiology, vol. 17, no. 3, pp. 261276, 2007.

[4] Y. Nakayama, N. Nakamura, D. Tsuji, K. Itoh, and A. Kurosaka, "Genetic diseases associated with protein glycosylation disorders in mammals," in Genetic Disorders, InTechOpen, 2013.

[5] http://www.stelic.com/cn27/pg122.html.

[6] N. Taniguchi, A. Suzuki, Y. Ito et al., Experimental Glycoscience: Glycobiology, Springer, New York, NY, USA, 2009.

[7] M. Janot, A. Audfray, C. Loriol, A. Germot, A. Maftah, and F. Dupuy, "Glycogenome expression dynamics during mouse $\mathrm{C} 2 \mathrm{C} 12$ myoblast differentiation suggests a sequential reorganization of membrane glycoconjugates," BMC Genomics, vol. 10, no. 483, 2009.

[8] Z. Zhang, J. Sun, L. Hao, C. Liu, H. Ma, and L. Jia, "Modification of glycosylation mediates the invasive properties of murine hepatocarcinoma cell lines to lymph nodes," PLoS ONE, vol. 20, no. 8, Article ID e65218, 2013.

[9] S. Ngamukote, M. Yanagisawa, T. Ariga, S. Ando, and R. K. Yu, "Developmental changes of glycosphingolipids and expression of glycogenes in mouse brains," Journal of Neurochemistry, vol. 103, no. 6, pp. 2327-2341, 2007.

[10] A. Urita, T. Matsuhashi, T. Onodera et al., "Alterations of high-mannose type $\mathrm{N}$-glycosylation in human and mouse osteoarthritis cartilage," Arthritis and Rheumatism, vol. 63, no. 11, pp. 3428-3438, 2011.

[11] C. Saravanan, Z. Cao, S. R. Head, and N. Panjwani, "Analysis of differential expression of glycosyltransferases in healing corneas by glycogene microarrays," Glycobiology, vol. 20, no. 1, pp. 13-23, 2010.

[12] C. Saravanan, Z. Cao, S. R. Head, and N. Panjwani, "Detection of differentially expressed wound-healing- related glycogenes in galectin-3-deficient mice," Investigative Ophthalmology and Visual Science, vol. 50, no. 12, pp. 5690-5696, 2009.

[13] K. A. Maupin, A. Sinha, E. Eugster et al., "Glycogene expression alterations associated with pancreatic cancer Epithelialmesenchymal transition in complementary model systems," PLoS ONE, vol. 5, no. 9, Article ID e13002, 2010.

[14] H. Ma, X. Miao, Q. Ma, W. Zheng, H. Zhou, and L. Jia, "Functional roles of glycogene and N-glycan in multidrug resistance of human breast cancer cells," IUBMB Life, vol. 65, no. 5, pp. 409-422, 2013.

[15] R. Guo, L. Cheng, Y. Zhao et al., "Glycogenes mediate the invasive properties and chemosensitivity of human hepatocarcinoma cells," The International Journal of Biochemistry and Cell Biology, vol. 45, no. 2, pp. 347-358, 2013.

[16] S. Diskin, J. Kumar, Z. Cao et al., "Detection of differentially expressed glycogenes in trabecular meshwork of eyes with 
primary open-angle glaucoma," Investigative Ophthalmology and Visual Science, vol. 47, no. 4, pp. 1491-1499, 2006.

[17] T. Inoue, H. Sugiyama, M. Kitagawa et al., "Abnormalities of glycogenes in tonsillar lymphocytes in IgA nephropathy," Advances in Otorhinolaryngology, vol. 72, pp. 71-74, 2011.

[18] T. Inoue, H. Sugiyama, Y. Hiki et al., "Differential expression of glycogenes in tonsillar B lymphocytes in association with proteinuria and renal dysfunction in IgA nephropathy," Clinical Immunology, vol. 136, no. 3, pp. 447-455, 2010.

[19] R. A. Kroes, G. Dawson, and J. R. Moskal, "Focused microarray analysis of glyco-gene expression in human glioblastomas.," Journal of neurochemistry, vol. 103, supplement s1, pp. 14-24, 2007.

[20] Z. Tan, W. Lu, X. Li et al., "Altered N-glycan expression profile in epithelial-to-mesenchymal transition of NMuMG cells revealed by an integrated strategy using mass spectrometry and glycogene and lectin microarray analysis," Journal of Proteome Research, vol. 13, no. 6, pp. 2783-2795, 2014.

[21] A. Mortazavi, B. A. Williams, K. McCue, L. Schaeffer, and B. Wold, "Mapping and quantifying mammalian transcriptomes by RNA-Seq," Nature Methods, vol. 5, no. 7, pp. 621-628, 2008.

[22] Y. Guo, Q. Sheng, J. Li, F. Ye, D. C. Samuels, and Y. Shyr, "Large scale comparison of gene expression levels by microarrays and RNAseq using TCGA data," PLoS ONE, vol. 8, no. 8, Article ID e71462, 2013.

[23] E. J. Lee, A. Malik, S. Pokharel et al., "Identification of genes differentially expressed in myogenin knock-down bovine muscle satellite cells during differentiation through RNA sequencing analysis," PLoS ONE, vol. 9, no. 3, Article ID e92447, 2014.

[24] http://www.uniprot.org/.

[25] Y. Moriya, M. Itoh, S. Okuda, A. C. Yoshizawa, and M. Kanehisa, "KAAS: an automatic genome annotation and pathway reconstruction server," Nucleic Acids Research, vol. 35, no. 2, supplement, pp. W182-W185, 2007.

[26] J. Montojo, K. Zuberi, H. Rodriguez et al., "GeneMANIA cytoscape plugin: fast gene function predictions on the desktop," Bioinformatics, vol. 26, no. 22, Article ID btq562, pp. 2927-2928, 2010.

[27] R. Albert, "Scale-free networks in cell biology," Journal of Cell Science, vol. 118, no. 21, pp. 4947-4957, 2005.

[28] X. R. Wu, Y. Zhu, and Y. Li, "Analyzing protein interaction networks via random graph model," International Journal of Information Technology, vol. 11, pp. 125-132, 2005.

[29] G. Su, A. Kuchinsky, J. H. Morris, D. J. States, and F. Meng, "GLay: community structure analysis of biological networks," Bioinformatics, vol. 26, no. 24, pp. 3135-3137, 2010.

[30] D. W. Farley, S. L. Donaldson, O. Comes et al., "The GeneMANIA prediction server: biological network integration for gene prioritization and predicting gene function," Nucleic Acids Research, vol. 38, no. 2, pp. W214-W220, 2010.

[31] M. E. Smoot, K. Ono, J. Ruscheinski, P. Wang, and T. Ideker, "Cytoscape 2.8: new features for data integration and network visualization," Bioinformatics, vol. 27, no. 3, pp. 431-432, 2011.

[32] B. Adamczyk, T. Tharmalingam, and P. M. Rudd, "Glycans as cancer biomarkers," Biochimica et Biophysica Acta-General Subjects, vol. 1820, no. 9, pp. 1347-1353, 2012.

[33] R. Barone, L. Sturiale, A. Palmigiano, M. Zappia, and D. Garozzo, "Glycomics of pediatric and adulthood diseases of the central nervous system," Journal of Proteomics, vol. 75, no. 17, pp. 5123-5139, 2012.
[34] Y. Sato and T. Endo, "Alteration of brain glycoproteins during aging," Geriatrics and Gerontology International, vol. 10, pp. S32-S40, 2010.

[35] D. D. W. Cornelison, S. A. Wilcox-Adelman, P. F. Goetinck, H. Rauvala, A. C. Rapraeger, and B. B. Olwin, "Essential and separable roles for Syndecan-3 and Syndecan-4 in skeletal muscle development and regeneration," Genes and Development, vol.18, no. 18, pp. 2231-2236, 2004.

[36] P. Dinh, C. Sotiriou, and M. J. Piccart, "The evolution of treatment strategies: aiming at the target," Breast, vol. 16, supplement 2, pp. 10-16, 2007.

[37] A. J. Nauta, N. Raashou-Jensen, A. Roos et al., "Mannosebinding lectin engagement with late apoptotic and necrotic cells," European Journal of Immunology, vol. 33, no. 10, pp. $2853-$ $2863,2003$.

[38] M. Møller-Kristensen, S. Thiel, A. Sjöholm, M. Matsushita, and J. C. Jensenius, "Cooperation between MASP-1 and MASP-2 in the generation of $\mathrm{C} 3$ convertase through the MBL pathway," International Immunology, vol. 19, no. 2, pp. 141-149, 2007.

[39] Y. Qian, I. Lee, W. Lee et al., "Functions of the $\alpha, \beta$, and $\gamma$ subunits of UDP-GlcNAc:Lysosomal enzyme $N$-acetylglucosamine-1-phosphotransferase," The Journal of Biological Chemistry, vol. 285, no. 5, pp. 3360-3370, 2010.

[40] B. Kuhn, J. Benz, M. Greif, A. M. Engel, H. Sobek, and M. G. Rudolph, "The structure of human $\alpha$-2, 6-sialyltransferase reveals the binding mode of complex glycans," Acta Crystallographica D, vol. 69, pp. 1826-1838, 2013.

[41] X. Bai, D. Zhou, J. R. Brown, B. E. Crawford, T. Hennet, and J. D. Esko, "Biosynthesis of the linkage region of glycosaminoglycans: cloning and activity of galactosyltransferase II, the sixth member of the $\beta 1,3$-galactosyltransferase family ( $\beta 3 \mathrm{GalT} 6)$," Journal of Biological Chemistry, vol. 276, no. 51, pp. 48189-48195, 2001.

[42] K. Uusi-Rauva, A. Kyttälä, R. Van Der Kant et al., "Neuronal ceroid lipofuscinosis protein CLN3 interacts with motor proteins and modifies location of late endosomal compartments," Cellular and Molecular Life Sciences, vol. 69, no. 12, pp. 20752089, 2012.

[43] Q. Xiao, F. Zhang, B. A. Nacev, J. O. Liu, and D. Pei, "Protein Nterminal processing: substrate specificity of Escherichia coli and human methionine aminopeptidases," Biochemistry, vol. 49, no. 26, pp. 5588-5599, 2010.

[44] J. Chen-Roetling, W. Liu, and R. F. Regan, "Hemopexin decreases hemin accumulation and catabolism by neural cells," Neurochemistry International, vol. 60, no. 5, pp. 488-494, 2012.

[45] E. Tolosano, S. Fagoonee, N. Morello, F. Vinchi, and V. Fiorito, "Heme scavenging and the other facets of hemopexin," Antioxidants \& Redox Signaling, vol. 12, no. 2, pp. 305-320, 2010.

[46] L. Camborieux, N. Bertrand, and J. P. Swerts, "Changes in expression and localization of hemopexin and its transcripts in injured nervous system: a comparison of central and peripheral tissues," Neuroscience, vol. 82, no. 4, pp. 1039-1052, 1997.

[47] R. C. Hunt, D. M. Hunt, N. Gaur, and A. Smith, "Hemopexin in the human retina: protection of the retina against hememediated toxicity," Journal of Cellular Physiology, vol. 168, no. 1, pp. 71-80, 1996.

[48] J. J. Kapojos, A. van Den Berg, H. van Goor et al., "Production of hemopexin by TNF- $\alpha$ stimulated human mesangial cells," Kidney International, vol. 63, no. 5, pp. 1681-1686, 2003.

[49] R.-C. Li, S. Saleem, G. Zhen et al., "Hemehemopexin complex attenuates neuronal cell death and stroke damage," Journal of 
Cerebral Blood Flow \& Metabolism, vol. 29, no. 5, pp. 953-964, 2009.

[50] M. Bechtel, M. V. Keller, W. Bloch et al., "Different domains in nidogen-1 and nidogen-2 drive basement membrane formation in skin organotypic cocultures," The FASEB Journal, vol. 26, no. 9, pp. 3637-3648, 2012.

[51] E. Kohfeldt, T. Sasaki, W. Göhring, and R. Timpl, "Nidogen2: a new basement membrane protein with diverse binding properties," Journal of Molecular Biology, vol. 282, no. 1, pp. 99109, 1998.

[52] M. Murshed, N. Smyth, N. Miosge et al., "The absence of nidogen 1 does not affect murine basement membrane formation," Molecular and Cellular Biology, vol. 20, no. 18, pp. 7007-7012, 2000.

[53] K. Salmivirta, J. F. Talts, M. Olsson, T. Sasaki, R. Timpl, and P. Ekblom, "Binding of mouse nidogen-2 to basement membrane components and cells and its expression in embryonic and adult tissues suggest complementary functions of the two nidogens," Experimental Cell Research, vol. 279, no. 2, pp. 188-201, 2002.

[54] J. W. Fox, U. Mayer, R. Nischt et al., "Recombinant nidogen consists of three globular domains and mediates binding of laminin to collagen type IV," EMBO Journal, vol. 10, no. 11, pp. 3137-3146, 1991.

[55] M. S. P. Ho, K. Böse, S. Mokkapati, R. Nischt, and N. Smyth, "Nidogens-extracellular matrix linker molecules," Microscopy Research and Technique, vol. 71, no. 5, pp. 387-395, 2008.

[56] S. Chakravarti, M. F. Tam, and A. E. Chung, "The basement membrane glycoprotein entactin promotes cell attachment and binds calcium ions," The Journal of Biological Chemistry, vol. 265, no. 18, pp. 10597-10603, 1990.

[57] R. M. Senior, H. D. Gresham, G. L. Griffin, E. J. Brown, and A. E. Chung, "Entactin stimulates neutrophil adhesion and chemotaxis through interactions between its Arg-Gly-Asp (RGD) domain and the leukocyte response integrin," Journal of Clinical Investigation, vol. 90, no. 6, pp. 2251-2257, 1992.

[58] F. D. Yelian, N. A. Edgeworth, L.-J. Dong, A. E. Chung, and D. R. Armant, "Recombinant entactin promotes mouse primary trophoblast cell adhesion and migration through the Arg-GlyAsp (RGD) recognition sequence," Journal of Cell Biology, vol. 121, no. 4, pp. 923-929, 1993.

[59] R. F. Nicosia, S. V. Nicosia, and M. Smith, "Vascular endothelial growth factor, platelet-derived growth factor, and insulin-like growth factor-1 promote rat aortic angiogenesis in vitro," The American Journal of Pathology, vol. 145, no. 5, pp. 1023-1029, 1994.

[60] N. Kimura, T. Toyoshima, T. Kojima, and M. Shimane, "Entactin-2: a new member of basement membrane protein with high homology to entactin/nidogen," Experimental Cell Research, vol. 241, no. 1, pp. 36-45, 1998.

[61] R. Neu, S. Adams, and B. Munz, "Differential expression of entactin-1/nidogen-1 and entactin-2/nidogen-2 in myogenic differentiation," Differentiation, vol. 74, no. 9-10, pp. 573-582, 2006.

[62] F. S. Grünewald, A. E. Prota, A. Giese, and K. Ballmer-Hofer, "Structure-function analysis of VEGF receptor activation and the role of coreceptors in angiogenic signaling," Biochimica et Biophysica Acta: Proteins and Proteomics, vol. 1804, no. 3, pp. 567-580, 2010.

[63] B. I. Terman, M. E. Carrion, E. Kovacs, B. A. Rasmussen, R. L. Eddy, and T. B. Shows, "Identification of a new endothelial cell growth factor receptor tyrosine kinase," Oncogene, vol. 6, no. 9, pp. 1677-1683, 1991.
[64] M. Shibuya, S. Yamaguchi, A. Yamane et al., "Nucleotide sequence and expression of a novel human receptor-type tyrosine kinase gene (flt) closely relatd to the fms family," Oncogene, vol. 5, no. 4, pp. 519-524, 1990.

[65] K. Pajusola, O. Aprelikova, J. Korhonen et al., "FLT4 receptor tyrosine kinase contains seven immunoglobulin-like loops and is expressed in multiple human tissues and cell lines," Cancer Research, vol. 52, no. 20, pp. 5738-5743, 1992.

[66] G. Neufeld, O. Kessler, and Y. Herzog, "The interaction of neuropilin-1 and neurropilin-2 with tyrosine-kinase receptors for VEGF,' Advances in Experimental Medicine and Biology, vol. 5, no. 15, pp. 81-90, 2002.

[67] S. Tessler, P. Rockwell, D. Hicklin et al., "Heparin modulates the interaction of VEGF165 with soluble and cell associated flk-1 receptors," Journal of Biological Chemistry, vol. 269, no. 17, pp. 12456-12461, 1994.

[68] J. Nishi, T. Minamino, H. Miyauchi et al., "Vascular endothelial growth factor receptor-1 regulates postnatal angiogenesis through inhibition of the excessive activation of Akt," Circulation Research, vol. 103, no. 3, pp. 261-268, 2008.

[69] G.-H. Fong, J. Rossant, M. Gertsenstein, and M. L. Breitman, "Role of the Flt-1 receptor tyrosine kinase in regulating the assembly of vascular endothelium," Nature, vol. 376, no. 6535, pp. 66-70, 1995.

[70] G. Fong, L. Zhang, D. Bryce, and J. Peng, "Increased hemangioblast commitment, not vascular disorganization, is the primary defect in flt-1 knock-out mice," Development, vol. 126, no. 13, pp. 3015-3025, 1999.

[71] J. B. Kearney, C. A. Ambler, K.-A. Monaco, N. Johnson, R. G. Rapoport, and V. L. Bautch, "Vascular endothelial growth factor receptor Flt-1 negatively regulates developmental blood vessel formation by modulating endothelial cell division," Blood, vol. 99, no. 7, pp. 2397-2407, 2002.

[72] B. Gao, W. Jeong, and Z. Tian, "Liver: an organ with predominant innate immunity," Hepatology, vol. 47, no. 2, pp. 729-736, 2008.

[73] V. Bieghs and C. Trautwein, "The innate immune response during liver inflammation and metabolic disease," Trends in Immunology, vol. 34, no. 9, pp. 446-452, 2013.

[74] S. Charrasse, M. Causeret, F. Comunale, A. Bonet-Kerrache, and C. Gauthier-Rouvière, "Rho GTPases and cadherin-based cell adhesion in skeletal muscle development," Journal of Muscle Research and Cell Motility, vol. 24, no. 4-6, pp. 309-313, 2003.

[75] K. A. Knudsen, L. Myers, and S. A. McElwee, "A role for the $\mathrm{Ca}^{2+}$-dependent adhesion molecule, $\mathrm{N}$-Cadherin, in myoblast interaction during myogenesis," Experimental Cell Research, vol. 188, no. 2, pp. 175-184, 1990.

[76] R. K. Yu, Y. Nakatani, and M. Yanagisawa, "The role of glycosphingolipid metabolism in the developing brain," Journal of Lipid Research, vol. 50, pp. S440-S445, 2009.

[77] L. Svennerholm, K. Bostrom, P. Fredman, J. E. Månsson, B. Rosengren, and B. M. Rynmark, "Human brain gangliosides: developmental changes from early fetal stage to advanced age," Biochimica et Biophysica Acta, vol. 1005, no. 2, pp. 109-117, 1989.

[78] R. K. Yu, L. J. Macala, T. Taki, H. M. Weinfeld, and F. S. $\mathrm{Yu}$, "Developmental changes in ganglioside composition and synthesis in embryonic rat brain," Journal of Neurochemistry, vol. 50, no. 6, pp. 1825-1829, 1988. 

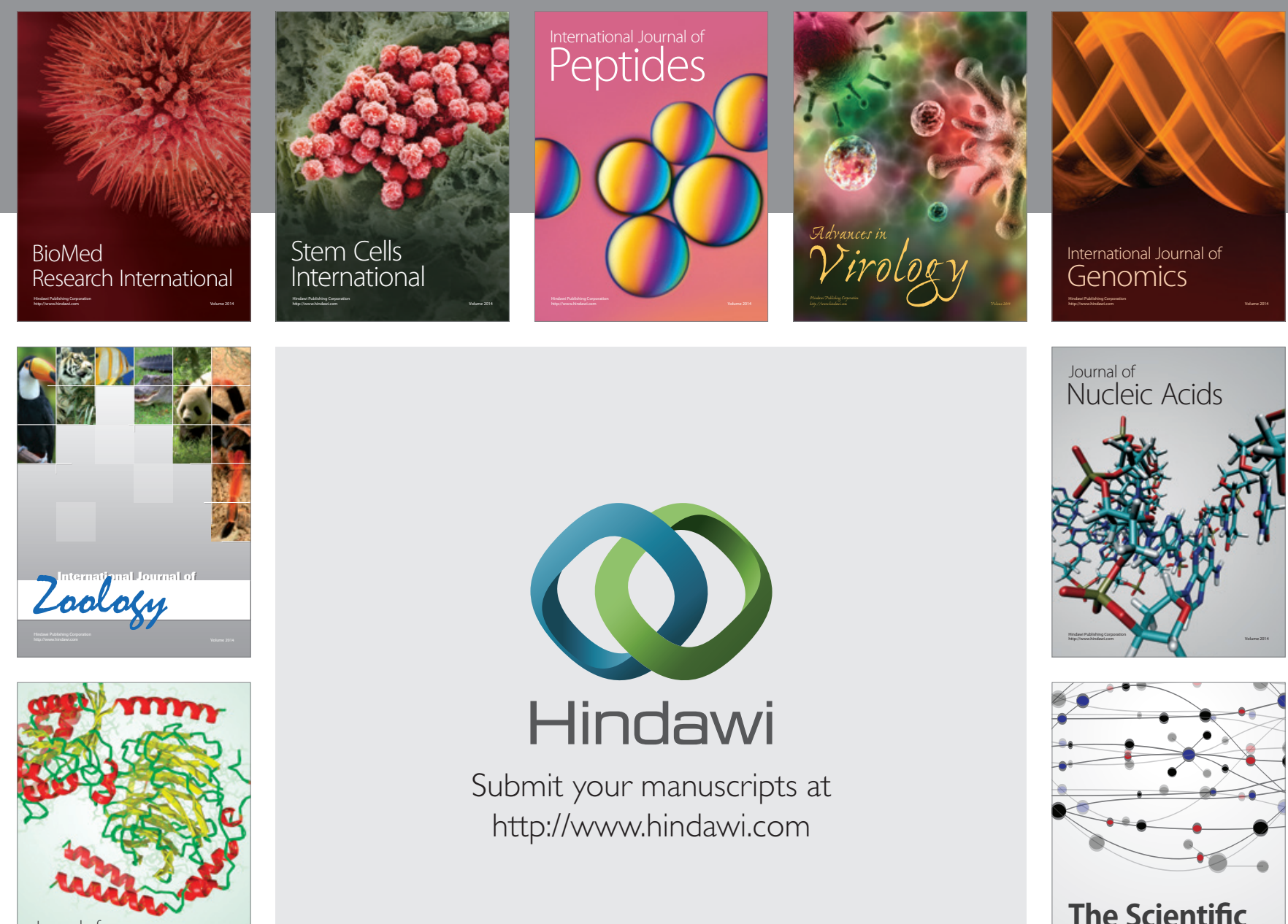

Submit your manuscripts at

http://www.hindawi.com

Journal of
Signal Transduction
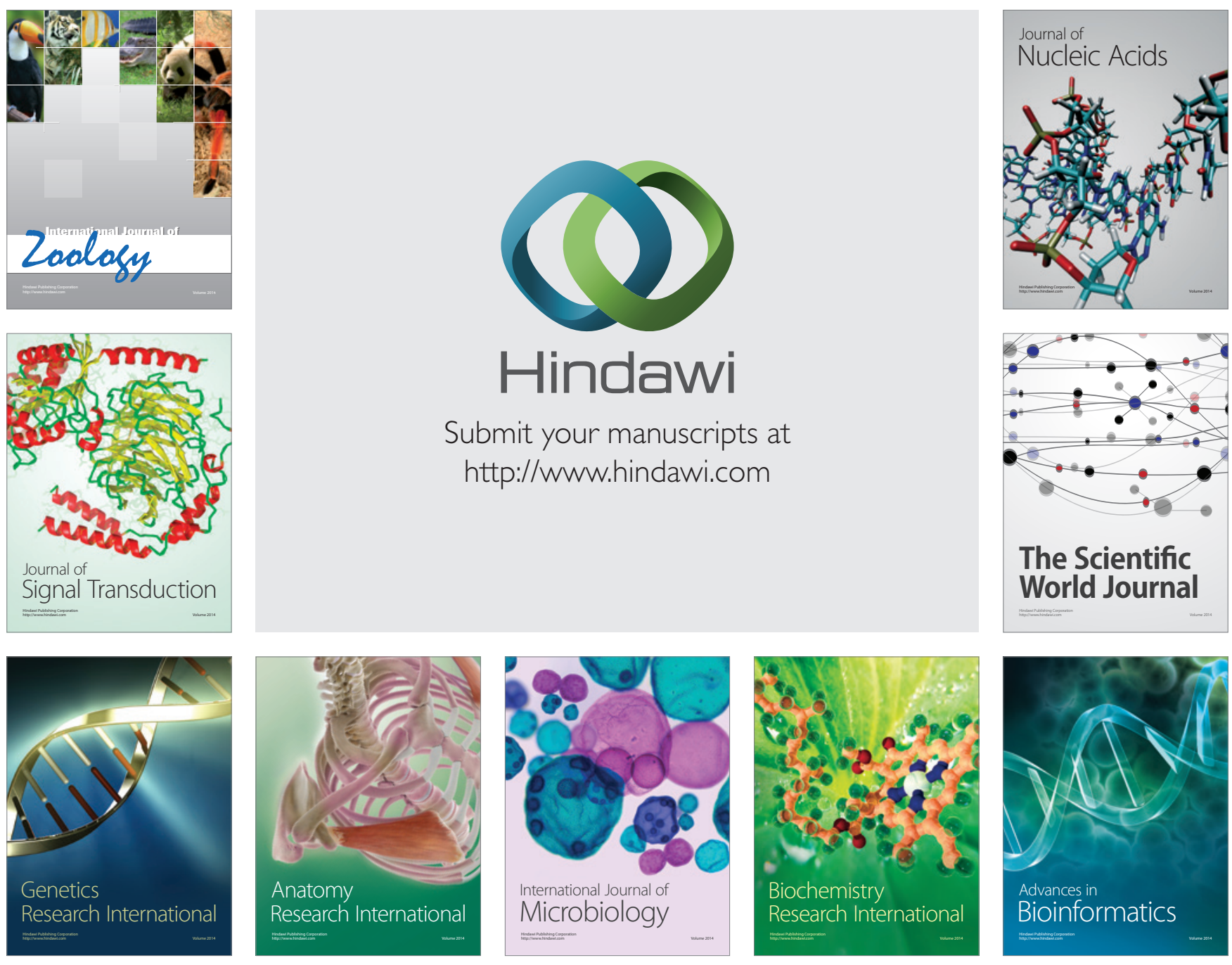

The Scientific World Journal
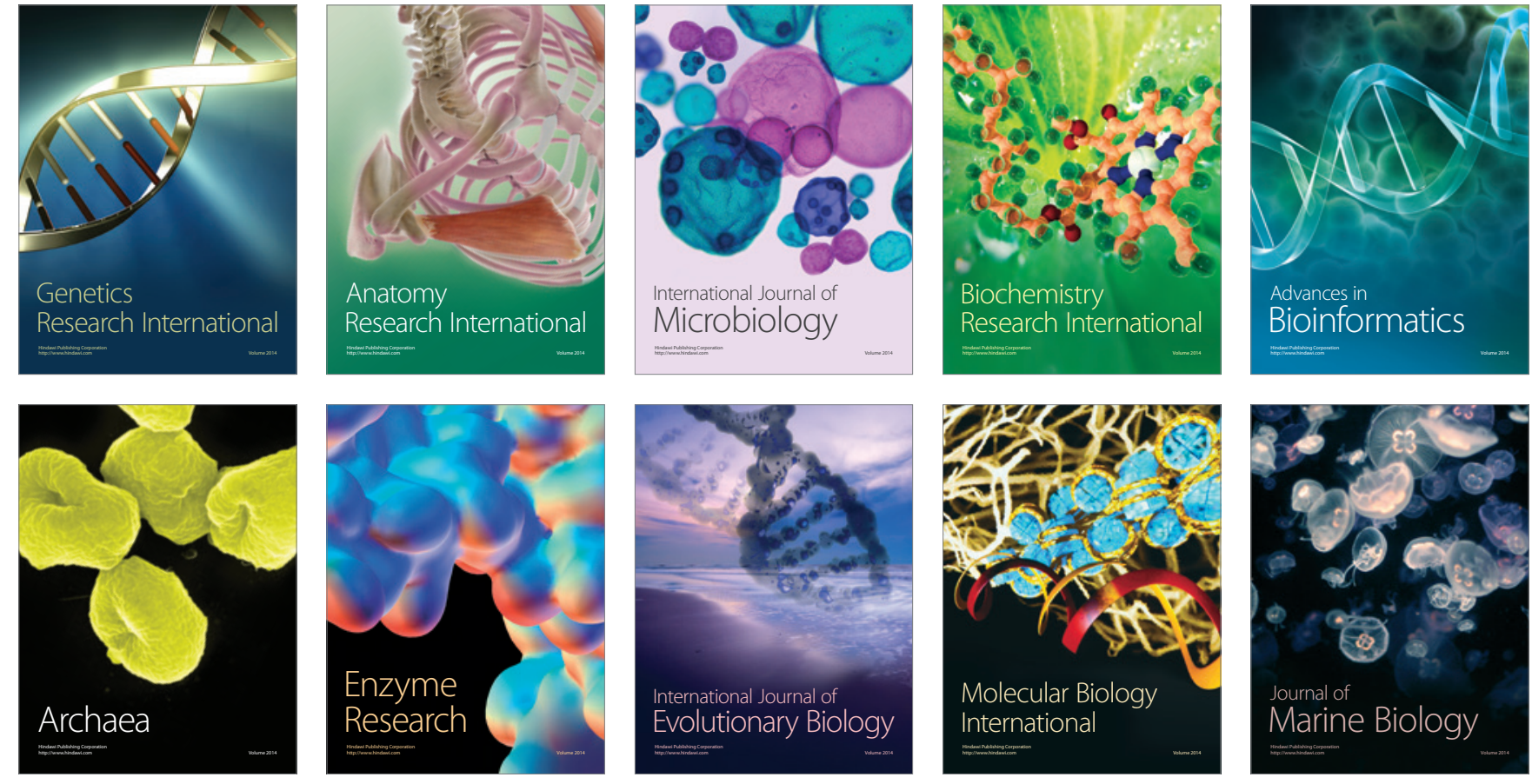